wenige Male einen kurzen Spaziergang ausführte, so wich doch die Lyspnoe niemals völlig wieder und auch die Kräfte kehrten nie in früherem Maasse zurück. Im Januar 1880 stellten sich die stundenlang anhaltenden Beängstigungen wieder ein, deren wir oben bereits gedachten und die Entwicklung einer beiderseitigen Brustwassersucht konnte nicht verkannt werden. Die Beine schwollen an, der Schlaf wich, das Athmen war nur in sitzender Stellung möglich. Es war eine Erlösung von schweren Leiden, die Wig gers mit Geduld und Ergebung getragen, als ihn der Tod am 23. Februar in der Morgenfrühe hinwegraffte.

Am 26. Februar wurde Wiggers Leiche unter einem zahlreichen Gefolge aus allen Kreisen der Universität und der Stadt Göttingen zur letzten Ruhestätte auf dem Albani-Friedhofe geleitet und in der Mitte seiner beiden, ihm im Tode vorausgegangenen Gattinnen beigesetzt. Dort ruhen die Reste eines hochverdienten und achtungswerthen M:nnes. Requiescant in pace!

Th. Husemann.

\title{
Ueber die Entstehung der Urochloralsäure und die Beschaffenheit der Chloralharne.
}

Von Dr. A. Bornträger in Marburg.

Bildung der Urochloralsäure im Organismus.

Bekanntlich hat Liebreich ${ }^{1}$ die vor einer Reihe von Jahren von ihm entdeckte hypnotische Wirkung des Chlorals auf eine Spaltung desselben im Blute in Chloroform und ameisensaures Salz zurückzuführen gesucht, indem er meinte, dass das freie Alkali des Blutes, unterstïtzt durch den oxydirenden Vorgang im Organismus, jene Zersetzung ebensowohl einleiten müsse, wie unverbundene Alkalien ausserhalb des letzteren.

Einen eigentlichen Beweis für seine Theorie hat Liebreich nicht geliefert, denn der von ihm gefundene Chlorgehalt der Expirationsluft chloralisirter Thiere muss nicht von Chloroformdampf herstammen, derselbe kann vielmehr, wie Hermann bemerkt

1) Berl. klin. Wochenschr. 1869. $325 \mathrm{ff}$, ferner: Das Chloralhydrat, ein neues Hypnoticum und Anaestheticum etc. O. Liebreich. Berlin 1871.

2) Experimentelle Toxicologie 1874. 272. 
hat, auch auf einen Gehalt an Chloral zurückgefuhrt werden, zumal Liebreich jenen Chlorgehalt nur nach Einführung eines bedeutenden Ueberschusses von Chloralhydrat in den Organismus beobachtete und Demarquay ${ }^{1}$ am Athem chloralisirter Thiere Chloralgeruch wahrgenommen haben will.

Aehnlich angestellte Versuche des Nachweises von Chloroform in der Expirationsluft chloralisirter Thiere ergaben Personne einen positiven, dagegen $\mathrm{Hammarsten},^{3} \mathrm{Rajewsky,} \mathrm{Frl.} \mathrm{To-}$ mascewicz ${ }^{5}$ und Musculns u. v. Mering ${ }^{6}$ negative Befunde.

Solche Widersprüche finden sich auch zwischen den Angaben verschiedener Forscher hinsichtlich des Nachweises eines Gehaltes des Blutes an Chloroform nach Einführung von Chloral in den Organismus. Personne und Richardson gelang derselbe, während Hammarsten und Rajewsky das Gegentheil berichten. Personne vermochte auch nachzuweisen, dass beim Erwärmen von Chloralhydrat mit Blut oder mit Hühnereiweiss anf $40^{\circ} \mathrm{C}$. Chloroform entsteht. Hinsichtlich des Blutes kam Hammarsten zu einem ähnlichen Resultate, doch konnte derselbe erst nach mehrstündiger Erwärmung diesen Vorgang constatiren. Der l'rocess verlief somit so langsam, dass nach Hermann's Ansicht der Chloroformgehalt des Blutes nach Chloralgenuss nur in ganz geringfugiger Weise die Wirkung des letzteren als solches unterstuitzen kann.

F al ck ${ }^{8}$ fand, dass eine einprocentige Kaliumhydratsolution in einer 10procentigen Chloralhydratlösung auch bei längerer Einwirkung in der Kälte keine Abscheidung von Chloroform mehr bewirkte, er bezeichnet daher die Annahme, das Alkali des Blutes sei im Stande einen solchen Effect zu äussern, als unerwiesen, zumal dasselbe nicht in unverbundenem Zustande, sondern in Form von Salzen vorbanden sei, deren zersetzende Kraft sicher viel geringer sei als diejenige der freien Basen. Dass einige Alkalisalze

1) Compt. rend, 69.640.

2) vide: Liebreich, Chloralhydrat pag. 23.

3) Upsala läkarefören. forehandl. 5. 424.

4) Centralbl. f. d. medic. Wissensch. 1870.211.

5) Pfüger's Archiv d. gesammten Physiologie 9. 35.

6) Berl. Chem. Berichte 1875. 662.

7) Compt. rend, 69. 980.

8) Zeitschr. f. pract. Medic. 1877. 247. 
thatsächlich eine Abspaltung von Chloroform aus Chloral bewirken, zeigen Versuche, welche Personne ${ }^{1}$ mit doppeltkohlensaurem, borsaurem und phosphorsaurem Natrinm angestellt hat.

Bei der Wiederholung dieser Versuche Falck's verwendete ich je 50 C.C. der Kaliumbydratsolutionen, denen ich nach der Erwärmung auf $37^{\circ} \mathrm{C}$. 0,5 g. krystallisirtes neutral reagirendes Chloralhydrat zusetzte.

In einer einprocentigen Kaliumhydratlösung lagerten sich nach einiger Zeit minimale Chloroformtröpfchen ab, die bei Bluttemperatur schon nach einer Stunde verschwanden, während der Chloroformgeruch bei nachherigem Stehen der Gemische in der Kälte nach nach 12 , nicht aber mehr nach 24 Stunden wahrgenommen werden konnte.

Bei Verwendung einer 0,5 procentigen Kalilange trat kein Chloroform mehr in Substanz, wohl aber deutlich dessen Geruch auf, der nach 24 Stunden kaum mehr zu erkennen war. Die Reaction der Flüssigkeit war zu dieser Zeit noch deutlich alkalisch.

0,2 und 0,1 procentige Kaliumhydratlösungen bewirkten eben. falls bald das Auftreten schwachen Chloroformgeruches, der im ersteren Falle nach 24 Stunden nur noch schwach, im letateren nicht mehr unterschieden werden konnte. In beiden Mischungen war die Anfangs ziemlich stark alkalische Reaction der neutralen gewichen.

Auch 0,05 und 0,02 procentige Kalilaugen bildeten bei einiger Daver der Einwirkung noch geringe Mengen Chloroform, die sich durch den Geruch unzweideutig zu erkennen gaben. Dieser verschwand bereits nach einer Stunde, während die Flüssigkeiten, von denen die erstere zu Anfang der Versuche Curcumapapier ziemlich stark, letztere nur schwach gebräunt hatte, bereits nach einer halben Stunde neutral reagirten.

Eine 0,01 procentige Kaliumhydratsolution, die auf Curcumapapier kaum mehr reagirte, zeigte sich Chloral gegenüber unwirksam.

Eine directe Vergleichung dieser Versuche mit den von Falck angegebenen ist nicht möglich, da Falck die eingehaltenen Verhältnisse nicht genau mitgetheilt hat, doch scheint mir der erste der von mir ausgeführten zu dem oben citirten von Falck in keinem

1) Compt. rend. 78.129.

Areh. A. Pharm. XVI. Bds. 6. Hft. 
erheblichen Widersprnche zu stehen. Dass ich auch bei Anwendung ganz schwacher Kalilösungen noch die Bildung geringer Quantitäten Chloroform mit Sicherheit nachzoweisen vermochte, scheint mir für die Richtigkeit der Untersuchungen von Personne und Richardson zu sprechen, bei welchen das Blut chloralisirter Thiere chloroformhaltig gefunden wurde, sowie der Angaben von Personne und $\mathrm{Hammarsten}$, dass bei Digestion von Blut mit Chloral Chloroform gebildet werde. Jedenfalls aber können die durch das freie Alkali des Blutes aus Chloral in oder ausserhalb des Organismus gebildeten Quantitäten Chloroform nicht gross gewesen sein und es erscheint Hermann's Ansicht gerechtfertigt, dass die Bildung unbedeutender Quantitäten Chloroform in der Blutbahn aus eingeführtem Chloral wohl denkbar sei, dass diese aber auf das Eintreten und den Verlauf des Chloralschlafes ohne nennenswerthen Einfluss bleiben dürften.

Auch bei den Untersuchungen von Chloralharnen wurden von den verschiedenen Forschern stark differirende Resultate erhalten. Während Liebreich im Harne chloralisirter Thiere eine Vermehrung der Chloride constatirte, giebt $\mathrm{Külz}^{1}$ an, Chloroform darin gefunden zu haben. Personne und Hammarsten vermochten nach Chloralisirungen weder Chloral noch Chloroform im Harne zu entdecken, wogegen Frl. Tomascewicz nach Darreichung von 4-6 g. Chloralhydrat im menschlichen Harne vermittelst der Hofmann'schen Isocyanphenylreaction regelmässig Chloral, aber kein Chloroform vorfand. Zum nämlichen Resultate kamen später Musculus und v. Mering. Falck vermochte im Urin einer Hündin nach Injection von $8,5 \mathrm{~g}$. Chloralhydrat in die Venen keine Spur desselben, ebensowenig Chloroform nachzuweisen.

Das Fehlen von Chloroform im Chloralharne kann nicht gegen Liebreich's Hypothese verwerthet werden, da in Betreff des Ueberganges des Chloroform's in den Harn die Angaben der einzelnen Autoren erheblich von einander abweichen. Hegar und Kaltenbach ${ }^{2}$ haben einen solchen bei Chloroformirungen constatiren können, ebenso Maréchal, ${ }^{3}$ dagegen Lall emand, Perrin und Duroy nicht. In Uebereinstimmung mit letzteren Autoren

1) Sitzungaber. d. Gesellsch. z. Beförd. d. Naturw. z. Marburg 1872. 38.

2) Virchow's Archiv. 49, $437 \mathrm{ff}$.

3) Pharmac. Centralhalle 1868. 362.

4) vide: Ziemssen, Pathologie u. Therapie. Bd.15. Intoxicationen. 1876. 122. 
vermochte ich in den Harnen dreier Kaninchen, welchen im Laufe eines Nachmittages je 1 C.C. $=1,48 \mathrm{~g}$. Chloroform (entsprechend 2,05 g. Chloralhydrat) subcutan injicirt wurde, dieses nicht nachzuweisen. Die Harne wurden einer zweistündigen Destillation aus dem Wasserbade unter Anwendung einer Kühlvorrichtung unterworfen, die Vorlagen, welche kein Destillat enthielten, mit alkoholischer Kalilösung ausgespült und diese nach Zusatz eines Tropfens Anilin gekocht. Da hierbei nicht der entfernteste Isonitrilgeruch auftrat, so war keine nennenswerthe Menge Chloroform in die Harne übergetreten, zumal Frl. Tomascewicz auf eine der beschriebenen sehr ähnliche Weise einen Zusatz von $0,03 \%$ Chloroform zu alkalischem und saurem Harne wiederzuerkennen vermochte.

Von hervorragendem Interesse für die Entscheidung der Frage über das Verhalten des Chlorals im Organismus ist die Entdeckung einer levogyren organischen säure im Chloralharne durch Musculus und v. Mering, ${ }^{1}$ welche Urochloralsäure genannt wurde. Die Autoren glauben, dass die Auffindung der Säure, welche sie isolirten und untersuchten, geeignet sei die Frage über die Chloralwirkung im thierischen Organismus endgültig zu entscheiden. Falck (l. c.) hat später ebenfalls das Auftreten der Urochloralsäure constatirt und deren Eigenschaften mit den von obigen Forschern angegebenen übereinstimmend gefunden. Auch Levinstein ${ }^{2}$ hat die linksdrehende Beschaffenheit der Chloralharne wahrgenommen.

Falck spricht am Ende seiner Mittheilungen die Erwartung aus, dass mit der genauen Kenntniss der Eigenschaften der Urochloralsäure die Aufrechterhaltung der Liebreich'schen Hypothese sich als unmöglich erweisen werde.

Obgleich Dragendorff ${ }^{3}$ die Abspaltung von Chloroform im Blute wegen der zu geringen Alkalinität des letzteren bezweifelt, so legt er dennoch die Idee an einen Verlauf des Processes sehr nahe, wobei zunächst $\mathrm{CHCl}^{3}$ und ameisensaures Salz gebildet würde und dann aus diesen Compenenten unter Zuziehung von Blut-

1) Bullet. de la Soc. Chim. 1874. 486 ; ferner: Berl. chem. Ber. 1875. 662.

2) Zur Pathologie der acuten Morphium - und Chloralvergiftung. Berl. klin. Woohenschr. 1876. No. 27.

3) Gerichtl. chem. Ermittel. von Giften. 1876. 36 n. 39. 
bestandtheilen die Urochloralsäure entstände, indem er nämlich die Möglichkeit ausspricht, dass die Urochloralsäure identisch sei mit einer Substanz, die nach Chloroformnarkosen im Harne gefunden werde mit der Fähigkeit Kupferoxyd in alkalischer Flüssigkeit wie Glucose zu reduciren, ohne deren anderweitige Reactionen zu theilen.

Dass diese Identität nicht besteht, mögen folgende Versuche zeigen.

Anmerkung: Ieh bemerke, dass die bei den folgenden 3 Versuchen untersuchten Harne vom Morgen des ersten Tages nach Ausführung der Injectionen dieselben waren, in welchen ich nach dem früher Mitgetheilten kein Chloroform entdecken konnte. Die käuflichen Injectionsspritzen enthalten nicht selten ein falsches Naass, ich habe deren zwar auch gefunden, wclche sehr annähernd 1 C.C. fassten, doch auch eine erhebliche Anzahl solcher, die nur 0,8 oder 0,9 C.C. Inhalt hatten.

\section{$\mathrm{v}$ ers u e h 1 .}

Einem Kaninchen wurde im Laufe eines Nachmittages 1 C.C. $=1,48 \mathrm{~g}$. Chloroform subcutan injicirt.

Die Menge des am folgenden Morgen vorgefundenen schwach alkalischen Urines betrug 95 C.C. Beim Versetzen der filtrirten Flüssigkeit mit Salpetersäure entstand sofort eine starke Trübung, welche beim Erhitzen in einen starken flockigen Niederschlag überging, somit von Serumeiweiss herrührte. Dies Auftreten von Eiweiss steht in Einklang mit den Angaben von $\mathrm{Hegar}$ und $\mathrm{Kal}$ tenbach, welche nach Chloroformirungen den Harn mehrmals albuminhaltig fanden.

Der durch Kochen mit Essigsäure von Albumin befreite Harn bewirkte vor und nach der Entfärbung durch Thierkohle eine ziemlich starke Reduction von Kupferoxyd, doch schieden sich erst nach einigem Stehen der gekochten Gemische geringe Mengen schlecht sedimentirenden Kupferoxydulhydrats aus. Die Waschwasser der verwendeten Kohlo bewirkten nur schwache Reductionen ohne nachfolgende Fällung von Oxydul oder dessen Hydrat. Ich bezeichne diese Art der Anstellung der Trommer'schen Probe in der Folge nach dem Erfinder als Seegen'sche ${ }^{1}$ Prïfungsmethode.

Da Seegen mit manchen concentrirten menschlichen Harnen ähnliche Reactionen erhalten hat, ohne aus 8-10 Litern eines

1) Seegen, Diabetes mellitus. 1875. 
derselben eine durch Polarisation oder Gährung erkennbare Znckermenge darstellen zu können und da Kaninchenbarn häufig solche Reductionserscheinungen ergiebt, so wäre der Schluss auf einen Zuckergehalt des Harnes nicht gerechtfertigt gewesen. Dass die Reduction auch nicht durch Urochloralsäure bewirkt wurde, folgt daraus, dass der nach dem Ansäuern mit Essigsänre durch Bleizuckerlösung entfärbte Urin optisch inactiv war. Die Prüfung wurde im Soleil-Ventzke'schen Diabetometer mit 0,1 Meter langer Beobachtungsröhre vorgenommen, dessen Gradtheilung direct Procente Glucose angiebt; auf dieses Instrument beziehen sich überhaupt alle in dieser Abhandlung gemachten Angaben uber Circumpolarisation.

Der am Morgen des zweiten Tages nach Ausführung der Injectionen in der Menge von 95 C.C. vorgefundene neutrale Harn enthielt ebenfalls Serumeiweiss, dessen Menge ich auf $0,3-0,4 \%$ scbätzte. Der von Albumin befreite Harn ergab bei Trommer's Probe schon vor Eintritt des Siedens eine höchst charakteristische, starke, orangegelbe Fällung, die über die Gegenwart von Zucker kaum einen Zweifel liess. Dem entsprechend zeigte das durch Versetzen von 20 C.C. des Harnes mit 1 C.C. Essigsäure und 2 C.C. Bleizuckerlösung erhaltene saure eiweissfreie Fluidum rechtsseitige Rotation von 0,45 Scalentheilen.

Der am dritten Morgen in der Menge von 24 C.C. vorgefundene Harn reagirte sauer, enthielt scheinbar ebensoviel Serumalbumin wie der des vorigen Tages und ergab bei der Prüfung nach Seegen schön ausgeprägte Reductionen. Der Zuckergehalt wurde optisch zu $0,66 \%$ gefunden; das Filtrat der Bleizuckerfällung war wiederum eiweissfrei. An diesem Tage starb das Thier.

\section{Versuch 2 .}

Da bei der Zersetzung des Chlorals durch Alkali auch ameisensaures Salz entsteht und es möglich erscheinen könnte, dass ohne dessen Mitwirkung die Urochloralsäure nicht entstehen könne, so habe ich bei den nunmehr folgenden Versuchen Kaninchen gleichzeitig Chloroform und ameisensaures Natrium in dem Gewichtsverhältnisse, in welchem diese Körper bei der Zersetzung von Chloralhydrat durch Aetznatron der Theorie nach entstehen, nämlich $0,842 \mathrm{~g}$. ameisensaures Natrium auf 1,48 g. (- 1 C.C.) 
Chloroform im Laufe eines Nachmittages injicirt; wobei die Canïlen der beiden Spritzen dicht neben einander eingeführt und letztere gleichzeitig entleert wurden.

Am folgenden Morgen wurden 90 C.C. alkalischen Urines angetroffen, der nur verschwindende Spuren von Albumin enthielt und bei Seegen's Probe nur Reactionen ergab wie normaler Kaninchenharn. Die Prüfung auf Urochloralsäure hatte ebenfalls ein negatives Ergebniss.

Am Nachmittage dieses Tages starb das Thier.

Versueh 3 .

Am ersten Morgen nach den Injectionen fand ich 23 C.C. stark alkalischen Urines mit ziemlich erheblichem Gehalte an Serumalbumin vor, der nach der Seegen'schen Probe zu urtheilen frei von einer nachweisbaren Menge reducirenden Zuckers war. Der in geeigneter Weise mit Bleizucker vorbereitete und dadurch auch eiweissfrei gewordene Harn war optisch inactiv, somit frei von Urochloralsäure.

Am zweiten Morgen 60 C.C. Urin, schwach alkalisch, starker Gehalt an Serumeiweiss.

Seegen's Probe:

a) Durch den von Albumin befreiten Harn starke Reduction, das entstandene Oxydul ging in Lösung (ich bezeichne diesen Vorgang von jetzt an als Farbenreaction), um sich erst nach einigem Stehen des gekochten Gemisches als Hydrat in Form eines starken, schlecht sedimentirenden Niederschlages wieder auszuscheiden.

b) Durch den mit Kohle entfärbten Harn starke Farbenreaction, bald darauf kräftige, leicht sich absetzende Füllung von Oxydulhydrat.

c) Durch das erste Waschwasser der Kohle nach sehr kurzem Kochen ziemlich bedeutender rother Niederschlag.

d) Durch das zweite schwache rothe Fällung.

e) Durch das dritte nur noch eine Spur $\mathrm{Cu}^{2} \mathrm{O}$.

f) Durch das vierte nur eine schwache Farbenreaction.

Der aus diesen Reactionen mit einiger Sicherheit zu folgernde geringe Gehalt des Harnes an Zucker konnte bei der Prüfung auf Urochloralsäure nicht in Betracht kommen, so dass sich aus 
der Inactivität des mit Bleizuckerlösung vorbereiteten und dadurch albuminfrei gewordenen Excretes das Fehlen jener Säure ergab.

Am dritten Morgen wurde neutraler Harn angetroffen, der auf Zusatz von Salpetersäure eine starke flockige Fällung ergab, die in der Wärme verschwand und in der Kälte sich wieder einstellte, ein Vorgang, der sich einigemal wiederholen liess. Essigsäure und Ferrocyankalium riefen ebenfalls einen kräftigen weissen Niederschlag hervor, ich hielt daher einen Gehalt des Harnes an Acidalbumin (Syntonin) für erwiesen. Diesen Körper fand bekanntlich Bence Jones ${ }^{1}$ zuerst im Harne eines Osteomalacischen, später ist dessen Auftreten auch im Urine nach starkem Morphiumgebrauch durch Levins te in ${ }^{2}$ constatirt worden.

Das durch Alkoholzusatz von Syntonin befreite, sodann stark eingeengte und wieder auf sein ursprüngliches Volum gebrachte Excret war frei von einer nachweisbaren Zuckermenge.

Am vierten Morgen wurde kein Harn vorgefunden; derjenige vom fünften entbielt nur eine geringe Spur Eiweiss and keinen Zucker. Der Harn vom sechsten Morgen war von beiden frei. An diesem Tage verendete das Kaninchen.

Resumé dieser 3 Thierversuche:

1) Weder Injectionen von $1,48 \mathrm{~g}$. Chloroform für sich, noch anch in Verbindung mit solchen von 0,842 g. ameisensaurem $\mathrm{Na}$ trium, wobei somit die Substanzen in dem Verhältnisse zu einander angewendet wurden, in welchem dieselben durch Natron aus 2,05 g. Chloralhydrat abgespalten werden, bedingen das Auftreten von Urochloralsäure im Harne.

2) Die Eiweissgehalte der Kaninchenharne waren nur bei dem ersten und dem dritten Versuche auf die Chloroforminjectionen zurückzuführen, während Reactionen, wie die beim zweiten Versuche beobachteten, häufig mit Excreten von Kaninchen erhalten werden. Bemerkenswerth ist das bei Versuch 3 beobachtete Auftreten von Acidalbumin.

3) Auch das Auftreten von Zucker im Urin wurde nur bei Versuch 1 und 3 constatirt und zwar in beiden Fällen erst in dem

1) Annal. d. Chem. u. Pharm. Bd. 67. 97-105.

2) Berl. klin. Wochenschr. 1877. 69.; ferner: Levinstein, Die Morphiumsucht. Berlin 1877. 96. 
am Morgen des zweiten Tages nach Ausführung der Injectionen angetroffenen Harne. Bei Versuoh 1 konnte der Zuckergehalt quantitativ durch optische Analyse ermittelt werden, bei Versuch 3 liess sich dies nicht bewerkstelligen, doch documentirte sich die Anwesenheit von Zucker in dem am zweiten Morgen nach den Injectionen angetroffenen Urine durch den Verlauf der Seegen'schen Probe mit einiger Sicherheit. Das Auftreten von Zucker im Urin nach Chloroforminhalationen ist bekanntlich wiederholt behauptet, aber auch häufig negirt worden. ${ }^{1}$ Maréchal ${ }^{2}$ hat gefunden, dass Harne nach Chloroforminhalationen Kupferoxyd reducirten, doch schrieb er dieses Verhalten deren Gehalt an Chloroform zu. Dass diese Auslegung auf unsere Fälle nicht passt, ergiebt sich aus dem schon erwähnten negativen Befunde der Prüfung der Harne auf Chloroform.

4) Die Serumalbumin enthaltenden Urine waren nach Entfärbung derselben durch Bleiacetat unter Ansäuerung mit Essigsäure stets albuminfrei, es empfiehlt sich daher Versuche darüber anzustellen, ob diese einfache Operation grössere Quantitäten Serumeiweiss, und auch die sonstigen bisweilen im Harne anzutreffenden Proteïnkörper zu beseitigen ermöglicht. Es könnte in diesem Falle die umstëndliche Coagulation des Albumins durch Essigsäure in der Siedhitze mit nachfolgender Abkühlung und Auffüllung auf das ursprüngliche Volum des Harnes umgangen werden.

Davon dass die Bildung der Urochloralsäure innerhalb der Blutbahn vor sich geht und ein Passiren des Chlorals durch den Magen nicht erforderlich ist, habe ich mich überzeugt, indem ich einem Kaninchen an einem Nachmittage $1 \mathrm{~g}$. Chloralhydrat in $3 \mathrm{C} . \mathrm{C}$. Wasser gelöst subcutan einspritzte und den am folgenden Morgen vorgefundenen alkalischen Urin urochloralsäurehaltig fand. Die Menge desselben betrug 45 C.C. Das durch Versetzen von 20 C.C. des Harnes mit 1 C.C. Essigsäure und 2 C.C. Bleizuckersolution erhaltene hellgelbe Flnidum rotirte $-1,45$. Auch der am Nachmittage in der Menge von 60 C.C. gelassene Harn rotirte noch stark, nämlich $-0,8$; der am folgenden Morgen vorgefundene war inactiv.

1) vide: Gmelin, Handbuch der Chem. 8. (1858) 388; ferner: Seegen, Diabetes mellitus. 1875 . 34 .

2) Pharm. Centralhalle. 1868. 362. 
Dass der Gehalt des Blutes an sogen. freiem Alkali allein nicht fähig ist bei der in thierischen Organismus herrschenden Temperatur Urochloralsäure aus Chloral zu erzengen, folgt daraus, dass ich bei keinem der Versuche über die Grenze der Verdünnung, bei welcher Kaliumhydrat noch Chloroform ans Chloral abzuspalten vermag, selbst bei 24 stündigem Erwärmen der Gөmische auf $36-37^{\circ} \mathrm{C}$. das Entstehen eines optisch activen Körpers constatiren konnte. Eine aus dem Aussehen des Harnes zu diagnosticirende vermehrte Auflösung von Hämoglobin im Blute findet bei Genuss von Chloralhydrat in hypnotischen Dosen für gewöhnlich nicht statt.

Da ich zu erfahren wünschte, ob die Bildung der Urochloralsäure durch die Blutbestandtheile anch ausserhalb des lebenden Organismus erfolgt, so erwärnte ich 3 Liter frisch entleerlen Schweineblutes auf $37^{\circ} \mathrm{C}$., setzte $2 \mathrm{~g}$. Chloralhydrat hinzu, und erhielt das Gemisch 24 Stunden auf jener Temperatur. Sodann verdünnte ich mit der doppelten Wassermenge, setzte Essigsäure zu und kochte, das entstandene voluminöse Coagulum wurde von der Flüssigkeit getrennt, abgepresst, mit Wasser zweimal ausgezogen und Filtrat sammt Waschwassern nochmals mit Essigsäure gekocht, wodurch abermals ein starkes, rothes Coagulum entstand. Eine Probe des nnnmehr resultirenden Filtrates von schwach rother Farbe ergab beim Kochen mit Essigsäure keine Trübung mehr, wohl aber mit Salpetersäure schon in der Kälte oine ziemlich starke. Um die Reste von Eiweiss und Farbstoff zu entfernen, wurde das ganze zuletzt erwähnte Fluidum mit einer geringen Quantität durch Salzsäure gereinigter Thierkohle eine Zeit lang gekocht und heiss filtrirt, wodurch ein farbloses, eiweissfreies Filtrat resultirte. Diese Fähigkeit der Thierkohle Eiweiss aufzunehmen hat $\mathrm{Cl}$. Bernard ${ }^{1}$ zuerst kundgegeben, später auch Levinstein. ${ }^{2}$ Die entfärbte Flïssigkeit wurde mit Kalilauge neutralisirt, auf ein geringes Volum eingedampft, sodann auf 120 C.C. gebracht und auf Polarisationsvermögen geprüft. Die Drehung betrug $-0,4$, dieselbe verschwand nicht durch neutrales, wohl aber durch basisches Bleiacetat, dieselbe konnte somit durch Urochloralsäure bedingt sein. Um über diesen Punkt Gewissheit

1) Archiv f. patholog. Anatomie 1856. 267.

2) Berliner klin. Wochensehr. 1877. 69. 
zu erhalten, verdampfte ich den Rest der Flüssigkeit (60 C.C.) zur Trockne, behandelte den Rückstand mit 90 procentigem Alkohol, verdampfte diesen und löste dessen Rückstand wieder in 60 C.C. Wasser. Die Lösung war optisch inactiv, es war somit obige Linksdrehung nicht durch urochloralsaures Kalium bedingt, denn dieses hätte sich in dem 90 procentigen Alkohol bei längerer Einwirkung wenigstens theilweise lösen müssen.

Ein zweiter in derselben Weise angestellter Versuch hatte gleichfalls ein negatives Resultat. Es ist mir somit nicht gelungen durch Digestion von Blut mit Chloralhydrat Urochloralsäure zu erhalten.

Die Entscheidung der Frage, ob das Chloral als solches oder erst nach der. Ueberführung in eine andere Substanz hypnotisch wirkt, ob z. B. die Urochloralsäure das ursächliche Moment des Chloralschlafes oder nur dasjenige Umwandlungsproduct ist, als welches das Chloral nach Aeusserung seiner hypnotischen Wirkung aus dem Organismus eliminirt wird, bleibt weiteren Untersuchungen ibberlassen. Bei solchen ist zu bedenken, dass aus einer bestimmten Menge Chloralhydrat im Organismus nach den Mittheilungen von Musculus und v. Mering und nach meinen Versuchen wenigstens die doppelte Quantität urochloralsauren Alkalis entsteht, da eine solche mit dem Harne ausgeschieden wird.

\section{Bestimmung der Urochloralsäure.}

Ein willkommener Anknüptungspunkt für die Auffindung einer Methode der Bestimmung der Urochloralsäure liegt in der Mittheilung von Musculus und v. Mering, dass die Säure linksseitiges Circumpolarisationsvermögen besitze, welches für das Kaliumsalz annähernd $-60^{\circ}$ betrage und von der Temperatur unabhängig zu sein scheine.

Um darüber zu entscheiden, ob die optische Bestimmung der Urochloralsäure im Harne statthaft ist, musste constatirt werden, ob die spec. Rotation derselben unabhängig ist:

a) Vom Procentgehalte der reinen wässerigen Lösungen ihrer Salze.

b) Von einem gleichzeitigen hohen Gehalte der letzteren an Harnbestandtheilen, wie Harnstoff, Alkalisalze etc.

c) $\mathrm{Ob}$ Momente, welche die genaue optische Untersuchung von Chloralharnen beeinträchtigen, wie dunkle Färbung oder 
schwer zu beseitigende Trübungen, auf einfache Weise beseitigt werden können, ohne dass dadurch eine Ausfällung oder Zersetzung von Urochloralsäure stattfindet und ohne dass sich das spec. Rotationsvermögen der letzteren ändert.

Bevor ich zur Erörterung dieser Fragen übergehe, will ich die Methode schildern, deren ich mich zur Darstellung des erforderlichen Materials bedient habe.

Darstellung des urochloralsauren Kaliums.

Dasselbe wurde nach einem von dem von Musculus und v. Mering eingehaltenen etwas abweichenden Verfahren bereitet. Ich nachte nämlich von der Fällbarkeit der Urochloralsäure durch basisches Bleiacetat Gebrauch, einer Eigenschaft der Säure, die jene Autoren zur Darstellung derselben wenig geeignet nannten. Chloralharne, deren Rotationen $-0,5$ überstiegen und welche, soweit sich dies beurtheilen lässt, frei von Zucker waren, wurden zunächst mit Bleizuckerlösung, sodann mit Bleiessig ausgefällt, der nach einigen Stunden von der Flüssigkeit getrennte Bleiessigniederschlag nach dem Auswaschen mit kaltem Wasser in solchem vertheilt und durch Schwefelwasserstoffgas zerlegt. Die erhaltene Lösung wurde nach Vertreibung des Schwefelwasserstoffs mit Kalilauge neutralisirt und nach mässigem Eindampfen nochmals mit neutralem, sodann wiederum mit basischem Bleiacetat ausgefällt. Die aus diesem zweiten Bleiessigniederschlage wieder in der angegebenen Weise erhaltene Solution von urochloralsaurem Kalium ergab beim Eindampfen einen hellbraunen Rückstand, der nach völliger Austrocknung auf dem Wasserbade zunächst mit reinem, sodann mit durch Salzsäure angesäuertem Aetheralkohol (2 Vol. Aether auf 1 Vol. Alkohol) wiederholt längere Zeit behandelt wurde. Die unter Zusatz von Säure gewonnenen Auszüge wurden nach der Neutralisation mit Kalilauge zur Trockene verdampft und der Rückstand nach dem Trocknen über Schwefelsäure mit absolutem Alkohol im Exsiccator zu wiederholten Malen längere Zeit behandelt. Letztere Procedur wurde mit Aether wiederholt und der Rückstand mit 90procentigem Alkohol extrahirt. Die so erhaltene Solution wurde mit viel Aether versetzt, wodurch sich in wenigen Minuten gelblichweisse feder- und büschelförmig gruppirte Krystalle des Kaliumsalzes abschieden, die abermals mit 90 procentigem Alkohol ausgezogen wurden. Das aus dieser Lösung wieder 
durch Aether gefällte weisse Präparat zeigte schönen Atlasglanz. Die Ausbente war eine sehr geringe, doch habe ich die beschriebene Art der Darstellung gewählt, weil mir dieselbe die Herstellung einer sehr annähernd reinen Substanz zu ermöglichen schien. Wegen der Schwierigkeit der Beschaffung einer grösseren Menge derselben habe ich eine Analyse nicht vorgenommen, indessen war dieselbe durch ihre Eigenschaften hinlänglich als das von $\mathrm{Muscu-}$ ins und v. Mering beschriebene urochloralsaure Kalium charakterisirt. Ich bemerke noch, dass die Darstellung sorgfältig mit dem Polariskop verfolgt wurde.

Ueber das specifische Rotationsvermögen des reinen urochloralsauren Kaliums in wässerigen Lösungen.

Bei Wiedergabe der einschlagenden Versuche sollen der Kürze halber stets nur die Mittel aus mehreren gut ïbereinstimmenden Zahlen angegeben werden, nicht diese selbst.

Eine Quantität des Kaliumsalzes in Wasser gelöst ergab eine Fliissigkeit mit der Rotation - 11,7, die nach 24 Stunden noch unverändert war, das Salz zei r.t somit keine Birotation. Bei Dilution der Lösung auf ihr dieifaches Volumen resultirte eine solche mit der bleibenden Rotation $-3,5$, von der 4 Vol. mit 3 Vol. Wasser verdïnnt eine Solution mit der bleibenden Drehung - 1,9 lieferten, die ihrerseits bei Verdünnung auf das doppelte Vol. die Rotation -- 0,95 annahm. Unter der Voraussetzung, dass das spec. Rotationsvermögen des urochloralsauren Kaliums in concentrirten und verdünnten Lösungen gleich gross wäre, hätten die Rotationen der aus der ursprünglichen Lösung bereiteten verdünnteren Flüssigkeiten $-3,9,-2,22$ und $-1,11$ betragen müssen, jene Uebereinstimmung besteht somit nicht. Indessen ist die spec. Rotation des Salzes in Lösungen mit Drehungen bis zu $-3,5$ nnd geringeren als nahezu constant zu erachten, wie daraus erhellt, dass die mit der Drehung - 3,5 behaftete Solution bei der Verdünnung mit Wasser im Verhältnisse $4: 3$ die Rotation - 1,9 statt - 2,0 und diese Flüssigkeit nach der Verdünnung auf ihr doppeltes Volum die Drehung - 0,95 statt der erwarteten von $-1,0$ zeigte.

Die aufgeführte Versuchsreihe wurde durch eine zweite bestätigt, indem eine Lösung des Kaliumsalzes mit der bleibenden Drehung -6,20 nach Dilution auf ihr 2-, 4- und 8-faches Vol. die 
Rotation - 2,8, $-1,50$ und $-0,70$ zeigte. Bei Constanz der spec. Rotation des Salzes hätten die Drehungen -3,10, - 1,55 und $-0,78$ beobachtet werden müssen. Ein Cinterschied in der spec. Rotation bei Lösungen mit der Drehung - 2,8 und geringeren liess sich nicht mehr wahrnehmen, indem statt der zu erwartenden Zahlen $-1,4$ und $-0,70$ die Werthe $-1,50$ und $-0,70$ erhalten wurden.

Es weicht somit die Urochloralsäure von der grösseren Zahl der optisch activen Substanzen ab, wie Rechtsweinsäure, ${ }^{1}$ Rohrzucker ${ }^{2}$ und circa 50 Substanzen $\left(\mathrm{Hesse}^{3}\right)$, für welche eine Abnahme der spec. Rotation bei steigendem Gehalte der Lösungen an activem Stoff beobachtet worden ist. Dagegen verhält sich nach Tollens 4 die Glucose analog der Urochloralsäure, ebenso der Campher nach Landolt. ${ }^{5}$

Da ich bei der Untersuchung einer grösseren Anzahl Morgenharne, bei allabendlicher Einnahme von 2 und $3 \mathrm{~g}$. Chloralhydrat, selbst bei hoher Concentration derselben, niemals eine 1,5 übersteigende Linksdrehung constatiren konnte und diesen Werth überdies nur einmal, so steht der polariskopischen Ermittelung der Urochloralsäure im Harne nach Darreichung von 2 und $3 \mathrm{~g}$. Chloralhydrat von Seiten der Veränderlichkeit ihrer spec. Rotation in Lösungen verschiedenen Procentgehaltes kein Hinderniss im Wege. Sollten Harne in Folge der Darreichung grösserer Dosen des Hypnoticums eine Linksdrehung zeigen, die 3,5 erreicht, so könnte auch in diesen die optische Prüfung noch direct vorgenommen werden, während bei noch stärkerer Rotation eine Verdünnung der Flüssigkeiten vor der polariskopischen Untersuchung vorgenommen werden müsste.

Bevor diese Methode angewendet werden kann, muss natïrlich erst die spec. Rotation der Urochloralsäure in einer Lösung

1) Biot, Mémoir. de l'Akadem. 15. 93 unl Arndtsen, Annal. de chim. et de phys. (3.) 54. 403.

2) Biot, Annal, de chim. et de phys. (3.) 36. 257 ; forner Tollens, Berliner chem. Ber. 1877. 1403 und Schmitz, eodem loco pag. 1414.

3) Annal. der Chem. u. Pharm. 176. 89, 189.

4) Berliner Chem. Berichte 1876. 1531.

5) Landolt, Das optische Drehungsvermögen organischer Substanzen. 1879. 81 . 
ermittelt werden, deren Drehung - 3,5 nicht übersteigt. Ich konnte aus Mangel an Material an diese Aufgabe nicht herantreten.

Ueber die Vorbereitung von Chloralharnen zur Polarisationsprobe, wenn deren Gehalte an Urochloralsäure ermittelt werden sollen.

Ich wende mich nunmehr der dritten der S. 426 aufgestellten Fragen zu, welche die Vorbereitung von Chloralharnen zur optischen Prüfung behandelt. Ich bespreche diese Frage vor der zweiten, weil bei Discutirung der letzteren Operationen erwähnt werden müssen, deren Zulässigkeit durch Versuche erwiesen wurde, die ich Behufs Beantwortung der dritten Frage angestellt habe.

Von den gebräuchlichsten Methoden zur Entfärbung dunkler Urine, nämlich:

a) Der Filtration durch animalische Kohle oder dem Behandeln oder Kochen mit solcher,

b) der Ausfällung der färbenden Materien durch neutrales und

c) durch basisches Bleiacetat

ist die letztere für unsere Zwecke wegen der Fällbarkeit der Urochloralsäure durch Bleiessig von vorneherein ausgeschlossen. Die Filtration von Harnen durch Thierkohle ist überhaupt bei quantitativen Untersuchungen gänzlich zu vermeiden, da dabei ein Verdunsten von Wasser vornehmlich in Sommer kaum zu vermei den ist.

Bei der Vorbereitung urochloralsäurehaltiger Harne zur optischen Untersuchung ist auch die Digestion mit Thierkohle unzulässig, da diese merkliche Quantitäten der Säure zurückzuhalten vermag, wie nachstehende Versuche zeigen.

\begin{tabular}{c|c|c}
\hline \multicolumn{2}{c|}{ Unveränderter Harn. } & Durch Kohle filtrirter Harn. \\
\hline Spec. Gew. & Rotation. & Drehung. \\
\hline & & $-0,80$ \\
1,035 & $-0,80$ & $-0,50$ \\
1,012 & $-0,45$ & $-0,50$ \\
1,028 & $-0,85$ & $-0,15$ \\
1,019 & $-0,70$ & $-0,65$ \\
& & $-0,20$
\end{tabular}


Um entscheiden za können, ob dic Entfärbung von Chloralharnen durch Bleizucker statthaft ist, musste fesigestellt werden, ob dieser in Harnen eine partielle Ausfällung von Urochloralsäure oder eine Steigerung der spec. Rotation derselben verursacht.

Eine Solution von urochloralsaurem Kalium mit der Rotation - 3,5 zeigte nach dem Versetzen mit einem genau gleichen Vol. kaltgesättigter Bleizuckerlösung die Drehung -2,15. Solutionen mit der Drehung - 1,90 und - 0,95 ergaben in derselben Weise behandelt Flüssigkeiten mit den Rotationen $-1,20$ und $-0,50$. Wäre in allen drei Fällen eine Steigerung des spec. Drehvermögens des uroohloralsauren Kaliums durch den starken Zusatz der Bleizuckerlösung nicht eingetreten, so hätten die Rotationen der Gemische $-1,75-0,95$ und $-0,475$ betragen müssen. Die erhaltenen Differenzen von $0,40,0,25$ und 0,025 zeigen, dass jene Steigerung $\mathrm{zwar}$ in Lösungen mit den Rotationen - 3,5 und -1,90, nicht aber mehr in solchen mit der Drehung $-0,95$ erfolgt. Die erwähnten Steigerungen waren keine vorübergehende, denn nach zwölfstündigem Stehen der klar gebliebenen Mischungen waren deren Rotationen noch unverändert.

In Uebereinstimmung mit diesen Versuchen stehen die folgenden, bei denen stets gleiche Vol. Harn und Bleiacetatlösung angewendet wurden. Der zuerst zu erwähnende Urin verdankte seine starke Rotation einem Zusatze von urochloralsaurem Kalium.

\begin{tabular}{c|c|c}
\hline Unveränderter Harn. & $\begin{array}{c}\text { Mit Bleizuckerlösung } \\
\text { vergetzter Harn. }\end{array}$ & $\begin{array}{c}\text { Bei Unwirksamkeit } \\
\text { des Bleizuckers zu } \\
\text { erwartende Drehun- } \\
\text { gen der Filtrate. }\end{array}$ \\
\hline Linksdrehung. & Linksdrehung. & \\
\hline 3,00 & 1,85 & $\mathbf{1 , 5 0}$ \\
1,50 & 0,75 & $\mathbf{0 , 7 5}$ \\
1,45 & 0,70 & $\mathbf{0 , 7 2 5}$ \\
1,35 & 0,70 & $\mathbf{0 , 6 7 5}$ \\
1,25 & 0,65 & 0,625 \\
1,10 & 0,60 & 0,55
\end{tabular}

Nach 24 stündigem Stehen der Filtrate waren diese noch klar und rotirten ebenso stark wie zu Beginn.

Die angeführten Versuche zeigen, dass eine Steigerung der spec. Rotation der Urochloralsäure oder eine partielle Ausfällung der letzteren bei Chloralharnen der Rotation $-\mathbf{1 , 5}$ selbst nicht 
durch Zusatz eines gleichen Volums starker Bleizuckerlösung erfolgt. Sollen Harne stärkerer Drehung zur optischen Untersuchung vorbereitet werden, so muss man suchen diesen Zweck mit Hülfe von möglichst wenig Bleiacetatsolution zu erreichen und sich dabei mit einer zweckentsprechenden Aufhellung begnügen.

Einige Versuche zur Begründung der Zulässigkeit der Aufhellung selbst stark rotirender Chloralharne mit Bleizucker führe ich unten an.

Nur nebenhin erwähne ich noch, dass ich mich der Ausfällung von Harnen mit Bleizucker, eventuell nach dem Ansäuern mit Essigsäure, auch häufig bedient habe, um trüb filtrirende Urine klar au erhalten, wobei der voluminöse Niederschlag der Bleiver. bindungen die feinvertheilten Stoffe einhüllte.

Ueber den Einfluss von Harnbestandtheilen auf das specifische Drehungsvermögen des urochloralsauren Kaliums.

Versuch 1.

Ein Chloralharn mit dor Rotation - 0,75 wurde über siedendem Wasserbade anf $1 / 5$ seines früheren Vol. eingeengt.

a) 10 C.C. davon wurden mit 40 C.C. Wasser versetzt, somit die ursprüngliche Concentration des Harnes wieder hergestellt. Drehung - 0,65 .

b) 10 C.C. des eingeengten Harnes wurden mit 10 C.C. Bleizuckersolution versetzt.

Drehung -1,80.

c) 10 C.C. des eingedampften Urines wurden mit 20 C.C. Bleiacetatlösung gefällt, Rotation -1,35.

$\mathrm{Zu}$ erwarten waren bei a, b und c die Rotationen - 0,75 , $-1,875$ und $-1,25$, es war somit keine Urochloralsäure beim Eindampfen des Urines zerstört worden, auch hatte der starke Zusatz der Bleizuckersolution zu dem eingeengten Harne weder eine Fällung der Säure, noch auch eine Steigerung der spec. Rotation derselben bewirkt.

Versuch 2.

Ein Chloralharn der Rotation - 0,45 wurde auf $1 / 6$ seines früheren Volums eingeengt. 
A. Bornträger, Ueher die Entstehung der Uroehloralsäure etc.

a) 20 C. C. der Flüssigkeit wurden mit 100 C. C. Wasser versetzt, somit wieder auf die ursprüngliche Concentration des Harnes gebracht.

\section{Drehung - 0,50.}

b) 20 C.C. des eingeengten Fluidurms mit 10 C.C. Bleiznckerlösung versetzt, rotirten $-1,85$ (erwartet $-1,80$ ). Dieser Versuch bestätigt das Resultat des ersten.

$$
\text { Versuch } 3 \text {. }
$$

Ein durch Thierkohle entfärbter und mit urochloralsaurem Kalium versetzter Harn rotirte $-1,20$.

250 C.C. davon auf 50 C.C. eingeengt ergaben eine Flüssigkeit von der Rotation $-6,0$.

a) 10 C.C. dieser Flüssigkeit wurden mit 40 C.C. Wasser versetzt, somit die anfängliche Concentration des Harnes wieder hergestellt.

$$
\text { Rotation }-1,30 \text {. }
$$

b) 20 C.C. wurden mit 10 C.C. Bleizuckerlösung versetzt, Rotation $-4,10$.

Auch bei diesem Versuche hatte eine Zersetzung von Urochloralsäure beim Eindampfen des Harnes nicht stattgefunden. $\mathrm{Ob}$ die starke Concentration des eingeengten Harnes dessen Rotation etwas herabgedrückt hatte, liess sich nicht sicher entscheiden, doch ist dies wahrscheinlich, da nach früher mitgetheilten Versuchen in einer Solution von urochloralsaurem Kalium mit der Rotation -6,0 das Salz ein höheres spec. Drehvermögen besitzt als in solchen mit der Drehung -1,20. Es würde in diesem Falle jene Verminderung dieser Steigerung das Gleichgewicht gehalten haben. Der Umstand, dass bei diesem Versuche ein Zusatz von 10 C.C. der Bleizuckerlösung zu 20 C.C. des eingedampften Harnes keine Steigerung der spec. Rotation der Urochloralsäure hervorrief, verbunden mit den entsprechenden Theilen der beiden vorhergehenden Versuche spricht für die Richtigkeit der pag. 432 ansgesprochenen Behauptung, dass bei einiger Vorsicht auch die Vorbereitung stärker als - 1,5 rotirender Chloralharne zur Polarisationsprobe mit Bleizucker vorgenommen werden könne. Das Resultat von $3 \mathrm{~b}$. steht in scheinbarem Widerspruche gegen früher mitgetheilte Versuche, indem in der urochloralsänrehaltigen Flüssigkeit mit der Drehung - 6,0 durch den Zusatz eines halben Vol. Bleizucker- 
lösung keine Steigerung der spec. Rotation der Säure eintrat, es ist indessen zu bemerken, dass es sich in diesem Falle nicht wie damals um einen Zusatz überschüssiger Bleisalzlösung handelte, denn ein solcher von 10 C.C. der Reagenslösung zu 20 C.C. des auf $1 / 5$ seines früheren Vol. eingeengten Harnes ist gleichwerthig demjenigen von 10 C.C. des Reagens zu 100 C.C. des unveränderten Excretes.

Die Bestimmung der Urochloralsäure in schwach drehenden Chloralharnen auf optischem Wege kann vorstehenden Versuchen zu Folge durch starke Einengung der Harne und Ausfällung der erhaltenen Flüssigkeiten durch Bleizuckerlösung erreicht werden. Dies Verfahren ist auch für Chloralharne mässiger Rotation z. B. $-0,50$ zu empfehlen, da bei deren directer Untersuchung etwa begangene Observationsfehler leicht einen erheblichen Theil der ganzen Drehung ausmachen können. Ich habe mich dieser abgeänderten Methode, wenn rathsam, bei den nachstehenden Versuchen stets bedient.

\section{Verlauf der Ausscheidung der Urochloralsäure mit dem Harn.}

Bei der Wiedergabe der folgenden Versuche werde ich von einer Mittheilung der Procent- und Gesammtgehalte der untersuchten Excrete an Urochloralsäure absehen und statt deren nur die beobachteten Rotationen resp. die Producte $X$ aus diesen und den Vol. der Urine angeben, wobei ich 100 C.C. als Volumeinheit setze. Jene Producte können leicht durch Multiplication mit dem Quotienten aus der spec. Rotation der Glucose und der Urochloralsäure in Gramme der letzteren umgerechnet werden, wenn deren spec. Rotation in weniger concentrirten Solutionen festgestellt sein wird.

Verlauf der Ausscheidung der Urochloralsäure mit dem Harn bei allabendlicher Einnabmeron 3 g. Chloralhydrat.

Es wurde gefunden, dass fast immer spät Abends vor erneuter Einnahme des Mittels noch urochloralsäurehaltiger Urin excernirt wurde. Die untersuchten Harne stammten von einem männlichen Individnum her, dessen zu verschiedenen Tageszeiten ausgeschiedene eiweiss - und allem Anscheine nach auch zuckerfreie Urine geson- 
A. Bornträger, Ueber die Entstchung der Urochloralsäure etc.

dert aufgefangen und mir überbracht wurden. Der allabendliche Gebrauch von Chloralhydrat in der Dosis von $3 \mathrm{~g}$. hatte vor Beginn der Versuche, von denen ich nur einige erwähne, schon ungefähr einen Monat lang stattgefunden.

1) 14. Aug. Abends $10 \mathrm{Uhr}$ vor erneuter Chloraleinnahme: 260 C.C. Harn. Drehung - 0,45.

$$
\mathrm{X}=\mathbf{1 , 1 7} \text {. }
$$

2) 15. Aug. duums.

An diesem Tage erhielt ich sämmtlichen Urin des Indivi-

a) Morgens $6 \mathrm{U}$.

$$
X=2,7 \text {, }
$$

d. h. in der Zeit vom 14. Abends $10 \mathrm{Uhr}$ bis zum 15. Morgens $6 \mathrm{Uhr}$ wurde der Harnblase eine Quantität Urochloralsäure zugeführt, die in der Vergleichszahl 2,7 einen Ausdruck fand, die während einer Stunde durchschnittlich übergetretene Menge wurde somit durch die Zahl $\mathbf{x}=\mathbf{0 , 3 3 7 5}$ repräsentirt.

b) Morgens 8 U. 30 .

$$
\begin{aligned}
& X=0,8313 \\
& x=0,3325 \\
& \text { Abends 10 U. (unmittelbar vor } \\
& \\
& \quad \begin{array}{l}
X=1,0238 \\
X=0,2048
\end{array}
\end{aligned}
$$

d) Abends $10 \mathrm{U}$. (unmittelbar vor erneuter Chloraleinnahme).

Für diesen Tag war somit:

$$
\Sigma X=5,6476
$$

3) 16. Aug. Auch an diesem Tage untersuchte ich sämmtlichen Urin des Mannes.
a) Morgens $6 \mathrm{U}$.
$\mathrm{X}=\mathbf{3 , 3 3 5 0}$
b) Vormittags $10 \mathrm{U} .30$.
$\mathrm{x}=\mathbf{0 , 4 1 6 9}$$$
\mathrm{X}=1,1550
$$$$
\mathrm{x}=\mathbf{0 , 2 5 6 7}
$$
c) Abends $10 \mathrm{U}$. (vor erneuter Chloraleinnahme).

$$
\begin{aligned}
& X=2,0286 \\
& x=0,1764
\end{aligned}
$$

Für diesen Tag war

$$
\Sigma X=6,5186
$$

4) 17. Aug.
a) Morgens $7 \mathrm{U}$.

$$
\begin{aligned}
& X=3,5000 \\
& X=0,3889
\end{aligned}
$$
b) Nachmittags $2 \mathrm{U}$.$$
\mathrm{X}=\mathbf{2 , 2 9 5 0}
$$$$
\mathrm{x}=\mathbf{0 , 3 2 7 9}
$$ 
A. Bornträger, Ueber dio Entstehung der Urocbloralaz̈ure etc.

c) Nachmittags $4 \mathrm{U}$.

$$
\begin{aligned}
& X=1,0000 \\
& X=0,5000
\end{aligned}
$$

d) Abends $6 \mathrm{U}$. wurde Urin entleert, den ich nicht erhielt.

e) Abend $10 \mathrm{U}$. (vor neuer Chloraleinnahme).

$$
\begin{aligned}
& X=0,8400 \\
& \mathbf{x}=\mathbf{0 , 2 1 0 0}
\end{aligned}
$$

Obgleich an diesem Tage eine Portion Harn verloren gegangen war, so fand ich doch $\Sigma X=\mathbf{7 , 6 3 5 0 ,}$, somit grösser als an den vorhergehenden Tagen, ein Zeichen, dass die Menge Urochloralsäure, die aus gleichen Quantitäten Chloral innerhalb eines Tages im Organismus entsteht und durch dic Nieren eliminirt wird, sehr variiren kann.

5) 20. Ang. Ich erhielt sämmtlichen Harn dieses Tages.

Die letzte Urinentleerung am vorhergegangenen Tage hatte A bends $10 \mathrm{Uhr}$ stattgefunden.
a) Morgens $6 \mathrm{U}$.
$\mathrm{X}=3,3825$
b) Nachmittags $4 \mathrm{U}$.
$\mathrm{x}=\mathbf{0 , 4 2 2 8}$
$X=4,0825$
$x=\mathbf{0 , 4 0 8 3}$

c) Abends $11 \mathrm{U}$. (vor abermaliger Chloraleinnahme).

$$
\begin{gathered}
X=2,0925 \\
x=\mathbf{0 , 2 9 8 9} \\
\Sigma X=\mathbf{9 , 5 5 7 5}
\end{gathered}
$$

6) 21. Aug.

a) Morgens $8 \mathrm{U}$. (erster
Harn dieses Tages).
$\mathrm{X}=3,7000$
b) Mittags $1 \mathrm{U}$.
$\mathrm{x}=\mathbf{0 , 4 1 1 1}$
$\mathrm{X}=\mathbf{1 , 7 5 0 0}$
$\mathbf{x}=\mathbf{0 , 3 5 0 0}$

c) Nachmittags $3 \mathrm{U}$.

$$
\begin{aligned}
& X=0,5177 \\
& X=0,2589
\end{aligned}
$$

d) Abends $6 \mathrm{U}$. wurde abermals Urin ausgeschieden, den ich aber nicht erhielt.

e) Abends 10 U. (vor erneuter Einnahme ron Chloralhydrat).

$$
\begin{aligned}
& X=0,9339 \\
& \mathbf{x}=\mathbf{0 , 2 3 3 5}
\end{aligned}
$$

$\boldsymbol{\Sigma X}$ an diesem Tage $=\mathbf{6 , 9 0 1 6}$, wobei natürlich der Harn von 6 Uhr Abends nicht berücksichtigt werden konnte. 
A. Bornträger, Ueber die Entstebung der Trochloralsäure etc.

7) 22. Aug.

a) Morgens $8 \mathrm{U}$. (erster

Harn).

$$
\mathrm{X}=4,5675
$$$$
\mathrm{x}=\mathbf{0 , 4 5 6 8}
$$
verloren.

c) Vormittags 12 U. 30. ging wieder eine Quantität Uंrin

d) Abends $6 \mathrm{U}$.

$\mathrm{X}=1,2000$

e) Abends 7 U. 30 .

$\mathbf{x}=\mathbf{0 , 2 1 8 2}$ b) Vormittags $11 \mathrm{U}$.

$$
\mathrm{X}=1,7500
$$

$x=0,5833$

f) Abends $10 \mathrm{Uhr}$ wurde wieder Urin excernirt, den ich nicht erhielt.

Obgleich somit an diesem Tage zwei Portionen Harn unberücksichtigt bleiben mussten, wurde doch $\Sigma \mathrm{X}$ zu $\mathbf{8 , 8 5 0 0}$ gefunden.

8) 24. Aug. Der letzte Urin vom vorhergehenden Tage war Abends $10 \mathrm{Uhr}$ gelassen worden.
a) Morgens $6 \mathrm{U}$.
$\mathrm{X}=2,3200$
b) Nachmittags $5 \mathrm{U}$.
$\mathbf{x}=\mathbf{0 , 2 9 0 0}$
$\mathrm{X}=2,6250$
$\mathrm{x}=\mathbf{0 , 2 3 8 6}$

c) Abends $10 \mathrm{U}$. (vor erneuter Chloraleinnahme).

Selbst in dem anf $1 / 10$ seines Vol. eingeengten, sodann mit Bleizuckerlösung versetzten Harne liess sich keine Rotation erkennen.

Auch an diesem Tage erhielt ich den gesammten Harn, $\mathbf{S X}$ war $=4,9450$.

Bei den soeben mitgetheilten Untersuchungen wurde gewöhnlich die grösste Menge Urochloralsäure pro Stunde während der Nacht der Harnblase zugefihrt und nahm diese Grösse vom Morgen nach dem Abende hin gradatim ab; indessen habe ich auch an einigen jener Versuchstage eine abweichende Beschaffenheit der Harne beobachtet, während nämlich die in der Zeit von Abends $10 \mathrm{Uhr}$ bis Morgens früh pro Stunde excernirten Mengen Urochloralsäure in den Zahlen:

$$
\begin{array}{r}
\mathrm{x}=0,3375 \\
0,4169 \\
0,3889 \\
0,4228 \\
0,4111 \\
0,4568 \\
0,2900
\end{array}
$$


einen Ausdruck fanden, die im Laufe des Tages ausgeschiedenen aber meistens in kleineren, wurde bei den Versuchen 4) c) und 7) b) und e) $\times$ zu 0,5000 resp. 0,5833 und 0,8883 gefunden, somit höhere Werthe als bei der Untersuchung der Frühharne bei den obigen Versuchen je erhalten wurden.

Beschaffenheit der Harne, wenn nach längerer allabendlicher Einnahme von 3 g. Chloralhydrat dessen Gebrauch sistirt wird.

Dass in solchen Fällen eine Ausscheidung von Urochloralsäure auch am zweiten Tage nach der letzten Einnahme ron Chloralhydrat noch stattfinden kann, zeigen folgende Untersuchungen.

1) Eine hysterische Frau erhielt nach einer längere Zeit fortgesetzten allabendlichen Darreichung obiger Dosis Chloralhydrat dieselbe zum letzten Male am 16. 5. Abends.

a) Gemischter Urin vom 17. 5. Abends und 18. 5. Morgens. Spec. Gew. 1,023, Rotation $-0,45$.

b) Gemischter Harn vom 18. 5. Abends und 19. 5. Morgens. Spec. Gew. 1,031, Rotation - 0,325.

Am Abende des 19. 5. erhielt die Kranke wieder Chloralhydrat, da das diesem substituirte Bromkalium niclit den gewünschten Erfolg hatte erreichen lassen. Die an den Versuchstagen zu verschiedenen Stunden excernirten Harne konnte ich nicht erhalten, sondern nur die vereinigten Abend- und Morgenurine; indessen zeigen obige Zahlen, dass ca. 48 Stunden nach der letzten Chloral-Einnahme noch Urochloralsäure in direct nachweisbarer Menge durch die Nieren ausgeschieden wurde.

Auch in einem zweiten Falle liess sich noch am zweiten Abende nach der letzten Einnahme von $3 \mathrm{~g}$. Chloralhydrat eine Ausscheidung von Urochloralsäure mit dem Harne erkennen.

Nach diesen Untersnchungen habe ich eine ziemlich grosse Anzahl von Versuchen über die Stärke des linksseitigen Rotationsvermögens der Frühharne bei allabendlicher Einnahme von 2, 3 und $4 \mathrm{~g}$. Chloralhydrat angestellt, da diese Dosen die gebräuchlichsten sind und die Morgenharne, wie oben erwähnt, gewöhnlich mehr Urochloralsäure enthalten als die später gelassenen.

Rotationen von Frühharnen bei allabendlicher Einnahme von $\boldsymbol{Z}$ g. Chloralhydrat.

Von 82 Urinen besassen 25 Rotationen von $-0,25$ und weniger, 21 solche von $-0,25$ bis $-0,40,14$ von $-0,4$ bis $-0,6$, 
9 von $-0,6$ bis $-0,8,9$ ron $-0,8$ bis $-1,0$ und 4 solche, welche diese Zahl überstiegen.

Diejenigen unter diesen Frühharnen, welche schwächer als $-0,4$ rotirten, besassen in der Regel ein spec. Gew., das 1,025 nicht überschritt, sich vielmehr gewöhnlich unter 1,020 hielt, indessen befanden sich unter den betreffenden 46 Harnen auch 4 , die ein spec. Gew. über 1,025, selbst bis zu 1,028 hatten.

Die 36 stärker als - 0,4 rotirenden Harne besassen meistens ein spec. Gew. über 1,025 bis zu 1,035 , und zwar wurde ein solches über 1,030 sehr häufig beobachtet.

Drehungender Morgenharne bei allabendlicher Einnahme von 3 g. Chloralhydrat.

Die Rotationen ron 70 bei allabendlicher Einnahme von $3 \mathrm{~g}$. Chloralhydrat gelassenen Frühbarnen variirten von Werthen, die nicht mehr direct ermittelt werden konnten, bis zu -1,25. 23 jener Harne zeigten Rotationen bis $-0,4$, wobei das spec. Gew. meistens unter 1,020 tlieb, 31 unter jenen 70 Urinen rotirten zwischen $-0,4$ und $-0,7$, darunter zeigten 8 spec. Gew. bis 1,020, 20 solche zwischen 1,020 und 1,030 und 3 ein 1,030 überschreitendes. 16 von jenen 70 Frühurinen zeigten Rotationen zwischen $-0,7$ und $-1,25$, diese hatten eämmtlich spec. Gew. über 1,020.

In Morgenurinen mittlerer Dichtigkeit (unter 1,020) ist demnach für gewöhnlich bei allabendlicher Einnahme von $3 \mathrm{~g}$. Chloralhydrat eine Rotation zu erwarten, welche von derjenigen der Frühharne nach Einnahme von 2 g. des Hypnoticums nicht erheblich abweicht.

Rotationen von Frähharnen bei allabendlicher Einnahme von 4 g. Chloralhydrat.

Ueber diesen Gegenstand habe ich nur eine geringe Anzahl von Versuchen angestellt, da die genannte Dosis in der Maison de Santé verhältnissmässig selten verabfolgt wurde.

Unter 21 untersuchten Harnen zeigten 14 Rotationen bis $-0,50$, ihre spec. Gew. blieben meistens unter 1,020. Von den übrig bleibenden $\gamma$ Harnen stärkerer Rotation sind nachstehend die Rotationen und spec. Gew. aufgeführt: 


\begin{tabular}{|c|c|c|c|c|c|}
\hline $\begin{array}{l}\text { Rotation. } \\
-1,35\end{array}$ & & & & & $\begin{array}{c}\text { spee. Gew. } \\
1,031\end{array}$ \\
\hline$-0,85$ & . & . & . & & . $\quad 1,024$ \\
\hline$-0,75$ & 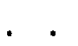 & . & $\cdot$ & & 1,031 \\
\hline$-0,75$ & . . & 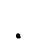 & . & & 1,027 \\
\hline$-0,75$ & . & • & $\cdot$ & & . $\quad 1,023$ \\
\hline$-0,65$ & . & . & $\cdot$ & & . $\quad 1,011$ \\
\hline$-0,55$ & • & & • & & 1,025 \\
\hline
\end{tabular}

Jedenfalls können die Morgenharne bei allabendlichem Genusse von $4 \mathrm{~g}$. Chloralhydrat auch stärkere Rotationen zeigen, da die vereinigten Abend - und Morgenharne läufig gleich stark und stärker rotirten, wie nachfolgende Zusammenstellung zeigt.

Hohe Rotationen combinirter Abend- und Morgenharne bei allabendlicher Einnahme von $4 \mathrm{~g}$. Chloralhydrat:

$\begin{array}{lll}-1,50 & -1,25 & -0,95 \\ -1,40 & -1,15 & -0,90 \\ -1,35 & -1,10 & -0,90 \\ -1,30 & -1,05 & -0,75 \\ -1,25 & -1,00 & \end{array}$

\section{Versuche fiber die Ausfallung der Urochloralsăure durch Bleiessig.}

Da ich häufig die Harne eines Diabetikers zu untersuchen hatte, der jeden Abend $3 \mathrm{~g}$. Chloralhydrat erhielt, so interessirte mich die Frage, wie die Eliminirung der Urochloralsäure aus Harnen zu ermöglichen sei, deren Zuckergehalte man durch Circumpolarisation zu ermitteln wünscht. Diese Frage ist bei der Untersuchung diabetischer Frïhharne geringen d. b. vom normalen wenig abweichenden Volums von Wichtigkeit, da bei diesen nach früher Mitgetheiltem bei Einführung von $3 \mathrm{~g}$. Chloralhydrat in den Organismus eine durch Urochloralsäuregehalt bedingte Linksdrehung bis zu 0,5 auftreten wird, die einen genan entsprechenden Antheil der Rechtsdrehung des Harnzuckers verdecken muss. Auch auf die Rotationen der 24 stündigen Quanta solcher Urine wird die aus $3 \mathrm{~g}$. Chloralhydrat innerhalb 24 Stunden entstehende Urochloralsäure noch merklich einwirken, dagegen wird bei den meisten diabetischen Harnen eine Beeinflussung der Rotation durch jene Säure wegen der erheblich vergrösserten Volume der Excrete sich kaum mehr geltend machen. 
Da nach Musculus und v. Mering basisch essigsanres Blei die Fähigkeit besitzt, die Urochloralsäure aus Urinen zu präcipitiren, so stellte ich Versuche an, nm in Erfahrung zu bringen, ob mit Hülfe jenes Salzes eine vollständige Ausfällung der Säure erzielt werden kann. Zu dem Ende wurden Chloralharne verschiedener Concentration und Drehung successive mit zunehmenden Quantitäten eines Bleiessigs vom spec. Gew. 1,3 versetzt und die erhaltenen Gemische theils nach kurzem, theils nach längerem Stehen derselben filtrirt. Der Kürze halber theile ich die angestellten Versuche in tabellarischer Anordnung mit, wobei ich das

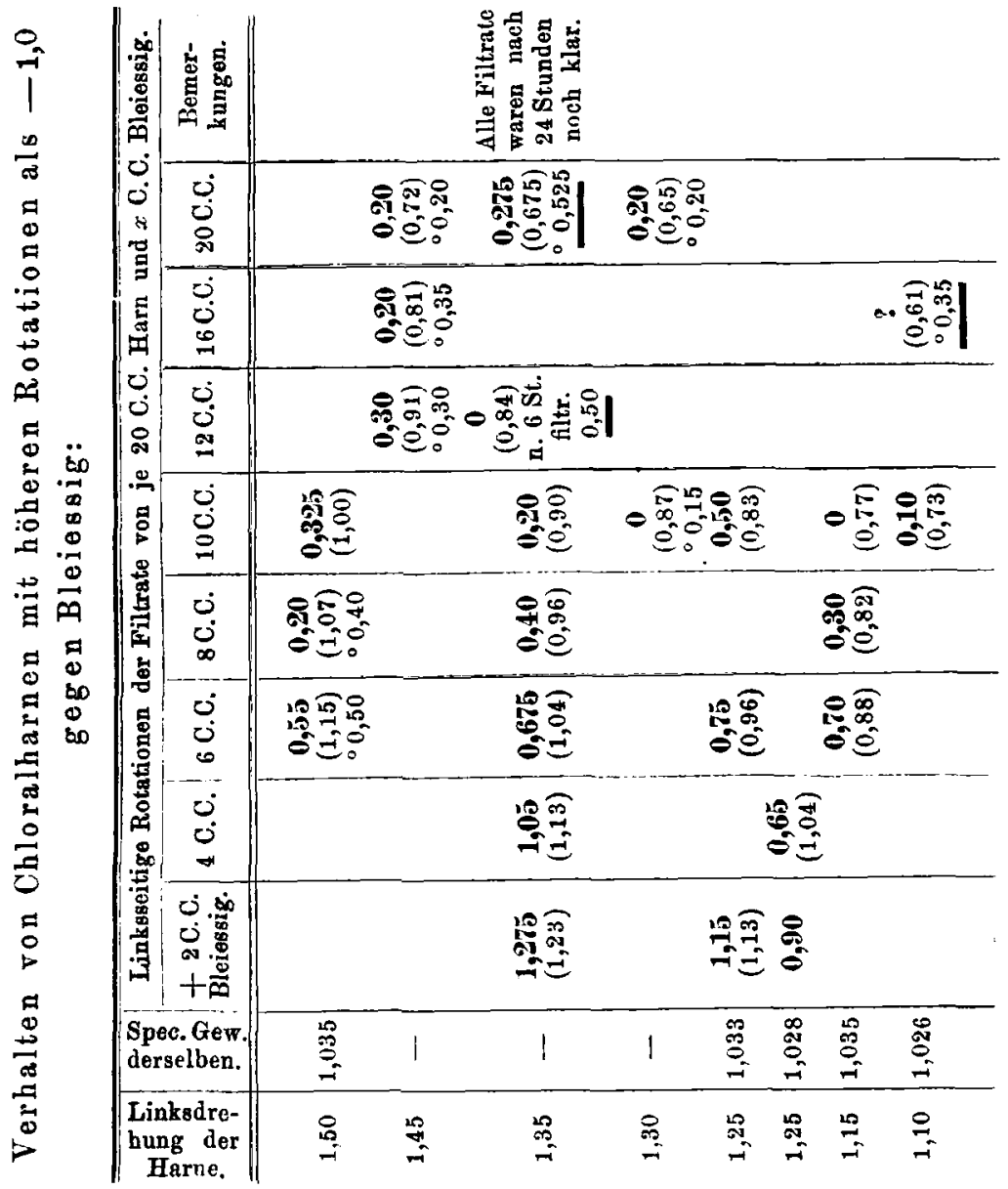


Minuszeichen stets fortlasse; die Zahlen, vor welchen kein Zeichen steht, kommen solchen Filtraten zu, die nach nur 5 Minuten währendem Stehen eines Theiles der Gemische erhalten wurden, während ein ${ }^{\circ}$ vor den Rotationen der nach eine Stunde währendem Stehen der Reste der nämlichen Mischungen resultirten Filtrate steht. Die eingeklammerten Zahlen drücken die Stärke der Rotationen aus, welche die Filtrate der Mischungen von Harn und Bleiessig hätten besitzen miissen, wenn letzterer keine Urochloralsäure ausfällte.

Bei einigen dieser Versuche ging bei längerem Stehen der mit überschüssigem basischem Bleiacetat versetzten Harne vor der Filtration ein Theil der Anfangs gefällten Urochloralsänre wieder in Lösung, wie sich aus der Vergleichung der Zahlen ergiebt, dic bei kürzerem und längerem Stehen der Mischungen vor der Filtration erhalten wurden. Dem entsprechend löste sich der in einer wässerigen Solntion von urochloralsaurem Kalium durch Bleiessig erzengte Niederschlag in einem Ueberschusse des Fällungsmittels wieder leicbt und rollständig aut'. Den Harnbestandtheilen kann auf Grund besonderer von mir angestellter Versuche ein solches Lösungsvermögen für jenen Niederschlag nicht beigemessen werden.

Da bei mehreren der nachfolgenden Versuche die nach einer Stunde filtrirten Proben schwächer rotirten als die nach 5 Minuten filtrirten, so ist ein längeres Stehenlassen der Filtrate anzurathen, um eine etwa eintretende Nachfällung von Urocbloralsäure constatiren zu können, dagegen ist ein längeres Stełienlassen der mit Bleiessig versetzten Harne vor der Filtration wegen der Wiederlöslichkeit der bereits gefällten Urochloralsäure im Ueberschusse des Reagens bei starkem Zusatz des letzteren nicht zu empfehlen.

Die zur Ausfällung einer bestimmten Menge Urochloralsäure aus gleichen Vol. verschiedener Harne erforderlichen Quantitäten Bleiessig differiren nach den nachfolgenden $V$ ersuchen nicht selten erheblich von einander, ein Umstand, der jedenfalls durch die variirende Beschaffenheit der Excrete bedingt ist. Während z. B. ein Zusatz von 2 C.C. Bleiessig zu 20 C.C. eines Harnes mit der Rotation - 0,70 und dem spec. Gew. 1,011 ein Sinken der Drehung auf $-0,25$ verursachte, blieb jencr Zusatz bei einem Urine von der Rotation $-0,675$ und dem spec. Gew. 1,029 ohne Einfluss anf die Drehung. Aehnliche Verschiedenheiten habe ich, wie 
A. Bornträger, Uober die Entstehung der Urochloralsäure etc.

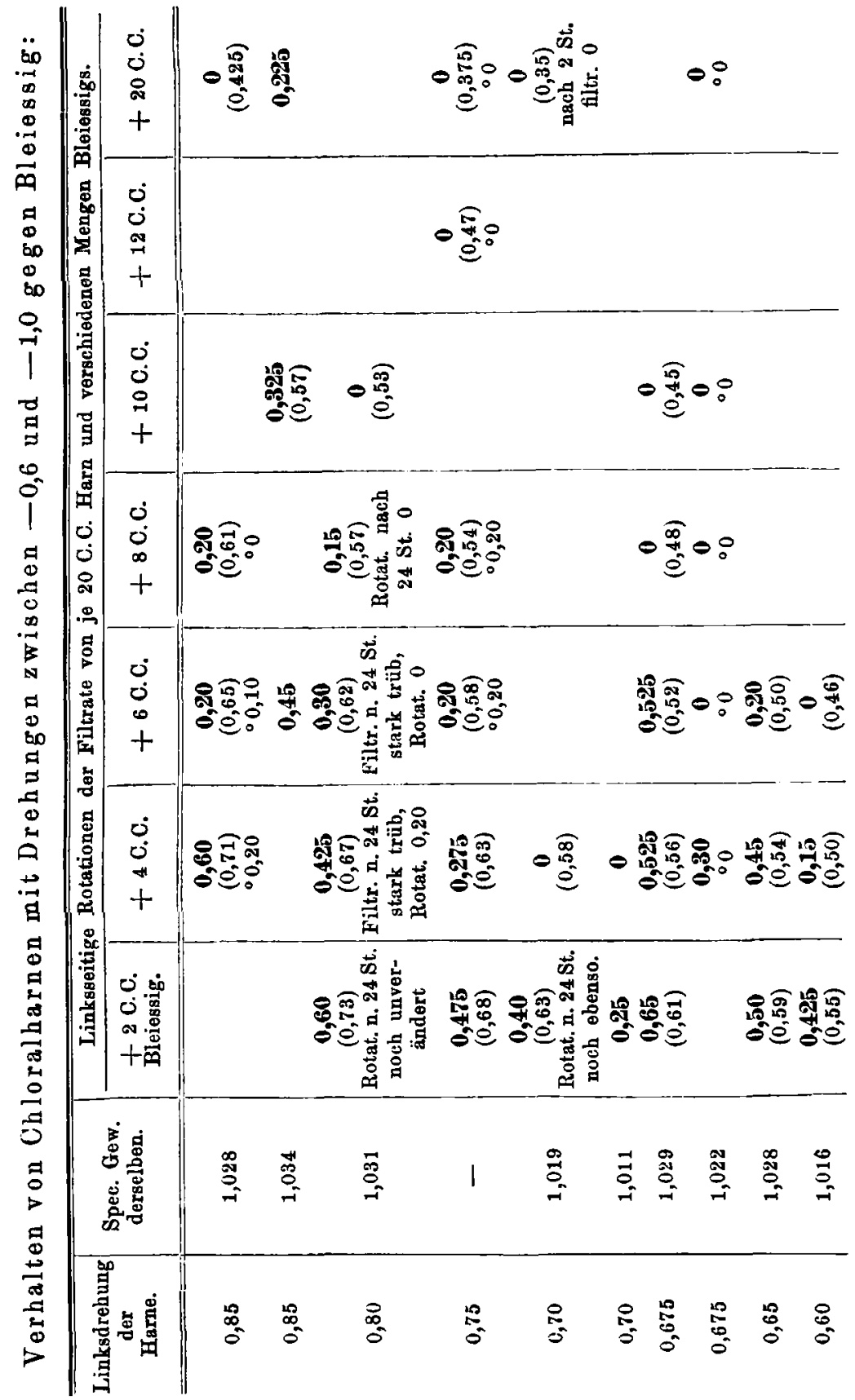


Verhalten von Chloralharnen mit Rotationen unter $-0,6$ gegen Bleiessig:

\begin{tabular}{|c|c|c|c|c|c|}
\hline \multirow{2}{*}{$\begin{array}{c}\text { Links- } \\
\text { drebung der } \\
\text { Harne. }\end{array}$} & \multirow{2}{*}{ Spec. Gew. } & \multicolumn{4}{|c|}{$\begin{array}{l}\text { Linksseitige Rotationen der Filtrate von je } 20 \mathrm{C.C} \text {. } \\
\text { Harn und verschiedenen Mengen Bleiessig. }\end{array}$} \\
\hline & & $\begin{array}{l}+2 \text { C. C. } \\
\text { Bleiessig. }\end{array}$ & +4 C.C. & + 6 C.0. & + 8 c.c. \\
\hline 0,50 & 1,019 & $\begin{array}{c}\mathbf{0 , 4 0} \\
(0,45)\end{array}$ & $\begin{array}{l}\mathbf{0 , 1 7 5} \\
(0,42)\end{array}$ & $\begin{array}{c}0 \\
(0,38)\end{array}$ & \\
\hline $\begin{array}{l}0,50 \\
0,50\end{array}$ & $\begin{array}{l}1,024 \\
1,020\end{array}$ & 0,40 & $\begin{array}{c}0,175 \\
0\end{array}$ & 0 & \\
\hline 0,475 & 1,022 & $\begin{array}{l}0,225 \\
(0,43)\end{array}$ & $\begin{array}{c}0 \\
(0,40)\end{array}$ & & \\
\hline $\begin{array}{l}0,475 \\
0,475 \\
0,475\end{array}$ & $\begin{array}{l}1,016 \\
1,016 \\
1,012\end{array}$ & $\begin{array}{c}0,20) \\
0 \\
0\end{array}$ & 0 & & \\
\hline 0,45 & - & $\begin{array}{c}\mathbf{0 , 3 5} \\
(0,41)\end{array}$ & $\begin{array}{c}\mathbf{0} \\
(0,38)\end{array}$ & & \\
\hline 0,45 & 一 & $\mathbf{0 , 3 0}$ & 0 & & \\
\hline 0,425 & 1,034 & $\begin{array}{c}\mathbf{0 , 4 0} \\
(0, \mathbf{3 9})\end{array}$ & $\begin{array}{l}\mathbf{0 , 3 7 5} \\
(0,35)\end{array}$ & $\begin{array}{c}0 \\
(0,33)\end{array}$ & \\
\hline 0,425 & 1,020 & 0,325 & 0 & & \\
\hline 0,425 & 1,019 & 0,325 & 0 & & \\
\hline $\begin{array}{l}0,425 \\
0,425\end{array}$ & $\begin{array}{c}1,012 \\
-\end{array}$ & $\begin{array}{l}0,30 \\
0,25\end{array}$ & $\begin{array}{l}0,10 \\
0,25\end{array}$ & $\mathbf{0}$ & \\
\hline 0,40 & 1,032 & 0,30 & $\begin{array}{c}0,30 \\
(0,33)\end{array}$ & & \\
\hline 0,40 & 1,030 & (0,20 & 0 & & \\
\hline 0,375 & 1,033 & $\begin{array}{c}0,20 \\
(0,34)\end{array}$ & $\stackrel{0}{(0.31)}$ & & \\
\hline 0,375 & 1,030 & 0,40 & 0,15 & 0 & \\
\hline 0,375 & 1,019 & 0,20 & 0 & & \\
\hline 0,375 & 1,028 & 0,325 & 0,225 & 0,175 & $\mathbf{0}$ \\
\hline 0,375 & 1,017 & 0,275 & 0 & & \\
\hline 0,375 & 1,015 & 0,125 & $\mathbf{0}$ & & \\
\hline 0,35 & 1,025 & $\begin{array}{c}0,30 \\
(0,32)\end{array}$ & & & \\
\hline 0,35 & 1,016 & $\mathbf{0 , 2 0}$ & $\mathbf{0}$ & & \\
\hline 0,30 & 1,040 & $\begin{array}{l}0,325 \\
(0,27)\end{array}$ & $\begin{array}{c}\mathbf{0 , 2 0} \\
(0,25)\end{array}$ & $\begin{array}{c}0,15 \\
(0,23)\end{array}$ & $\begin{array}{c}0 \\
(0,21)\end{array}$ \\
\hline 0,30 & 1,031 & 0,30 & & & \\
\hline 0,30 & 1,023 & 0 & & & \\
\hline 0,30 & 1,019 & 0 & & & \\
\hline 0,30 & 1,017 & 0,20 & 0 & & \\
\hline 0,30 & 1,016 & 0 & 0 & & \\
\hline 0,275 & 1,021 & $(0,25)$ & & & \\
\hline 0,275 & 1,016 & 0 & & & \\
\hline 0,25 & 1,032 & $\begin{array}{l}0,20 \\
(0,23)\end{array}$ & $\begin{array}{c}0 \\
(0.21)\end{array}$ & & \\
\hline 0,25 & 1,028 & 0 & 0 & & \\
\hline 0,25 & 1,017 & 0,25 & 0 & & \\
\hline 0,225 & 1,016 & $(0,20)$ & & & \\
\hline 0,225 & 1,014 & 0 & & & \\
\hline
\end{tabular}


aus obigen Tabellen ersichtlich, nicht selten beobachtet, ohne aber immer den Grund dafïr in erheblichen Abweichungen der Concentration oder der Färbung der untersuchten Urine finden zu können. Es ist somit nicht möglich bei der Ausfällung der Urochloralsäure ans Harnen sofort die richtige Menge Bleiessig zn treffen, es muissen vielmehr stets mehrere Versuche mit wachsenden Zusätzen des Reagens vorgenommen und die Prüfung kann erst dann als beendigt angesehen werden, wenn eine Steigerung des Bleiessigzusatzes die Rotation des Harnes nicht mehr stärker sinken macht als der Vermehrung des Flüssigkeitsquantums entspricht.

\section{Bestimmng des Zuckers in Harnen neben Urochloralsäure.}

Bei gleichzeitigem hohen Gehalte von Urinen an Zucker und Urochloralsäure ist von der beschriebenen Methode der Ausfüllung der Säure kein günstiger Erfolg zu erwarten, da grosse Quantitäten Bleiessig aus stark zuckerhaltigen Harnen einen nicht unerheblichen Theil des Zuckers präcipitiren, auf welchen Gegenstand ich später (p. 447 f.) zurückkommen werde. Bei der Untersuchung diabetischer Harne kommt dieser Umstand im Allgemeinen nicht in Betracht, da solche bekanntlich in der Regel von grossem Volum und hohem Zuckergehalt oder von mittlerem Vol. und mässigem resp. auch schwachem Zuckergehalte sind. Im ersteren Falle wird der Einfluss der Urochloralsäure auf die Rotation so gering sein, dass derselbe vernachlässigt oder durch mässige Quantitäten Bleiessig beseitigt werden kann, welche keine Ausfüllung von Zucker hervorrufen. Im zweiten Falle ist selbst von einem starken Bleiessigzusatz eine Präcipitation von Harnzucker kaum zu befürchten. Es bleiben somit nur noch die Fälle zu betrachten, in welchen es sich $\mathrm{um}$ die Untersuchung von Urinen bei Chloralintoxicationen handelt. Levinstein hat nach einer solchen durch 20-24 g. Chloralhydrat den Harn stark zuckerhaltig gefunden, dieser rotirte $+1,9$. Die Ausfällung mit Bleiessig hätte die genaue optische Bestimmung des Zuckers wahrscheinlich nicht ermöglichen lassen, ebensowenig die Fehling'sche 'Titrirmethode wegen der Fähigkeit der Urochloralsäure Kupferoxyd zu reduciren.

In etwa vorkommenden ähnlichen Fällen kann folgendes Verfahren zur Kenntniss der Gehalte von Harnen an Zucker und Crochloralsäure führen. 
Man ermittelt zunächst die Rotationen der Harne, überlässt diese mehrere Wochen sich selbst und bestimmt von Zeit zu Zeit deren Drehungen, welche schliesslich in Folge des Zerfalls des Zuckers bei der Gährung linksseitige sein und längere Zeit constant bleiben werden. Später wird auch die Urochloralsäure zersetzt. Aus den erhaltenen Maximalwerthen der Linksdrehungen und den anfänglichen Rotationen ergeben sich durch sehr einfache Rechnungen die Gehalte der untersuchten Excrete an Zucker und Urochloralsäure. Dieses Verfahren stuitzt sich auf einige Versuche, von denen ich nur den folgenden anführe, da die anderen ähnliche Resultate ergaben.

Von einem bereits trübe gewordenen diabetischen Harne mit der Rotation $+4,2$ wurden am 10. Sept. 100 C.C. mit 88,5 C.C. einer wässerigen Lösung von urochloralsaurem Kalium mit der Drehung - 4,7 versetzt, wodurch eine optisch inactive Mischung resultirte, die in einen engen Messcylinder gegeben und sich selbst überlassen wurde. Die unbedeutende Menge des zwischen zwoi Prüfungen derselben im Polarisknp verdunstenden Wassers wurde stets vor Anstellung der Polarisationsprobe ersetzt.

16. Sept. Rotation -0,50.

20. Sept. Rotation - 0,90. (Der Harn war stark sauer.)

24. Sept. Rotation $-2,10$.

Da den anfänglichen Gehalten der Mischung an Zucker und Urochloralsäure die Rotationen $\pm 2,23$ entsprachen, so war am 24. Sept. schon sämmtlicher Zucker zersetzt. Bei weiterem Stehen der Flüssigkeit verschwand deren linksseitiges Rotationsvermögen langsam vollständig; während nämlich der Zucker schon nach 2 Wochen vollständig zerfallen war, bedurfte die Urochloralsäure dazu mehr als eines Monates.

Dieses Verfahren, welches dem von Hoppe-Seyler ${ }^{1}$ zur Unterscheidung der durch Gallensäuren und durch Zucker bedingten Rechtsdrehungen von Harnen angegebenen ähnlich ist, allerdings setzt Hoppe-Seyler der zu untersuchenden Flüssigkeit Hefe zu, kann bei einer supponirten Vergiftung durch Chloral als eine Art von Bestätigung der ärztlichen Diagnose und der gerichtlich chemischen Untersuchung einen Gehalt des Harnes an linksdrehender Substanz, die Urochloralsäure sein kann, erkennen lassen.

1) Physiol. u. patholog. ehem. Anal, 1875, 339. 
Nachweis der Fällbarkeit von Zucker aus Harnen durch basisches Bleiacetat.

Auf Seite 445 sagte ich, dass grosse Quantitäten Bleiessigs aus stark zuckerhaltigen Urinen einen Theil des Kohlehydrates zu fällen vermögen. Diese Thatsache ist zuerst ron $\mathrm{Br}$ ücke ${ }^{1}$ bekannt gegeben worden, der eine partielle Ausfällung des Zuckers aus künstlichen und pathologischen Zuckerharnen erreichen konnte. Da Bleiessig in reinen Glucoselösungen keine Fällung zu Stande bringt, so schloss Brücke, dass eine Substanz im Harne enthalten sei, die die Fällbarkeit des Harnzuckers durch Bleiessig bedinge.

Gegen die Versuche Brizcke's hat Seegen ${ }^{2}$ eingewendet, dass die dort beschriebenen Reactionen nicht mit Bestimmtheit auf Rechnung von Zucker zu setzen seien, der durch Bleiessig gefällt worden sei, sondern dass dieselben auch von solchem ausgehen konnten, der dem ungenügend gereinigten Niederschlage der Bleiverbindungen nur mechanisch anhaftete. Bei dieser Argumentation hat Seegen übersehen, dass nach Brücke's Mittheilungen Wasser, welches mit dem abgepressten Bleiessigniederschlage in Berührung gewesen war, nur minimale Zuckerreactionen ergab, so dass Brücke's Schluss, dass ein Theil des Zuckers durch den Bleiessig präcipitirt worden sei, vollkommen gerechtfertigt erscheint.

Um ein Urtheil über den Grad der Fällbarkeit des Zuckers aus Urinen durch Bleiessig zu gewinnen, habe ich eine Anzahl von Versuchen mit diabetischen Harnen und Bleiessig angestellt, deren Ergebnisse ich in einer Tabelle zusammenfassen will. Zuvor bemerke ich noch, dass bei einem Versuche mit einer reinen 7,5 procentigen wässerigen Glucosesolution, höhere Zuckergehalte fand Seegen selten in diabetischen Harnen, durch starken Bleiessigzusatz, selbst eines gleichen Volums, das spec. Rotationsvermögen der Glucose in der klar gebliebenen Flüssigkeit nicht modificirt wurde. Nach 24 stündiger Aufbewahrung in wohlverschlossenem Gefässe zeigte die gelbgewordene Mischung eine Abnahme der Rotation von $+3,7$ bis $+3,0$ ohne dass ein Niederschlag entstanden war. Bei Anwendung von Bleizuckerlösung statt des Blei-

1) Sitzungsber, d. mathemat.-naturwissensch. Classe der Akadem. d. Wissensch. zu Wien. 39. 10 .

2) Sitzungsber. dor mathem.-naturwissensch. Classe der Akadem. d. Wissensch. zu Wien, Bd. 64. (Jahrgang 1871.) Abth. 2. 50. 
essigs trat eine Verminderung der Rotation der Mischung bei 24 stündigem Stehen nicht ein. Das zu diesen, wie zu allen anderen in dieser Abhandlung zu erwähnenden, Versuchen mit Glucose angewendete Präparat war nach Mohr's ${ }^{1}$ Vorschrift aus käuflicher rein weisser Glucose bereitet worden.

Die Gemische wurden stets nach 5 Minuten währendem Stehen filtrirt. In dieser Tabelle steht ein \# vor den Zahlen, welche die Rotationen der Filtrate nach 24 stündigem Stehen angeben.

\begin{tabular}{|c|c|c|c|c|c|c|c|c|}
\hline \multirow{2}{*}{ 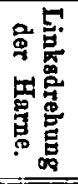 } & \multirow{2}{*}{ 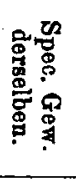 } & \multicolumn{7}{|c|}{$\begin{array}{l}\text { Linksdrehungen der Filtrate von } 20 \text { C. C. Harn und × C. C. } \\
\text { Bleiessig. }\end{array}$} \\
\hline & & $\begin{array}{l}\text { + } 2 \text { C.C. } \\
\text { Bleiessig. }\end{array}$ & $+4 \mathrm{Cc}$. & $+5 \mathrm{Cc}$ & $+6 \mathrm{Cc}$. & $+8 \mathrm{Cc}$ & $+10 \mathrm{Cc}$ & $+20 \mathrm{Cc}$ \\
\hline 7,00 & 1,035 & & & $\begin{array}{c}5,25 \\
(5,6) \\
+5,10\end{array}$ & & & $\begin{array}{c}4,20 \\
(4,6) \\
\text { n. 4 St. } \\
\text { fltt. } \\
4,15\end{array}$ & $\begin{array}{c}\text { N. } 4 \mathrm{St} \text {. } \\
\text { filtr. } \\
2,90 \\
(3,5)\end{array}$ \\
\hline 6,30 & 1,035 & $\begin{array}{r}5,60 \\
(5,7) \\
+5,6\end{array}$ & $\begin{array}{c}5,00 \\
(5,25) \\
+5,0\end{array}$ & & $\begin{array}{c}4,55 \\
(4,85) \\
+4,45\end{array}$ & & $\begin{array}{c}\mathbf{3 , 9 0} \\
(4,20) \\
+3,50\end{array}$ & $\begin{array}{c}2,95 \\
(3,15) \\
+2,20\end{array}$ \\
\hline 5,80 & - & & & & & $\begin{array}{r}3,90 \\
(4,15) \\
+3,80\end{array}$ & & \\
\hline 4,00 & 1,026 & & & $\begin{array}{c}3,00 \\
(3,2) \\
+2,95\end{array}$ & & & $\begin{array}{c}2,50 \\
(2,70) \\
\mathbb{H}^{2,35}\end{array}$ & $\begin{array}{c}2,10 \\
(2,0) \\
+1,20\end{array}$ \\
\hline 1,95 & 1,021 & & & $\begin{array}{c}1,45 \\
(1,55) \\
+1,35\end{array}$ & & & $\begin{array}{c}1,15 \\
(1,30) \\
+0,90\end{array}$ & $\begin{array}{c}1,00 \\
(1,0) \\
+0,70\end{array}$ \\
\hline 1,75 & 1,026 & & & $\begin{array}{c}1,40 \\
(1,40) \\
+1,45\end{array}$ & & & $\begin{array}{c}1,00 \\
(1,20) \\
+10,75\end{array}$ & $\begin{array}{c}0,70 \\
(0,90) \\
-0,30\end{array}$ \\
\hline 1,30 & - & $\begin{array}{c}1,25 \\
(1,18)\end{array}$ & & $\begin{array}{c}1,00 \\
(1,05) \\
+1,05\end{array}$ & & & $\begin{array}{c}0,80 \\
(0,87) \\
+0,75\end{array}$ & $\begin{array}{c}0,60 \\
(0,65) \\
+0,40\end{array}$ \\
\hline
\end{tabular}

Die beiden ersten Versuche bestätigen die Fällbarkeit eines Theiles des in Harnen enthaltenen Zuckers durch Bleiessig. In dem $4 \%$ Zucker enthaltenden Urine bewirkte selbst der Zusatz eines gleichen Vol. Bleiessig keine durch das Polariskop sicher nachweisbare Ausfällung von Zucker.

Der zweite Versuch zeigt, dass bei vorsichtiger Aufhellung zuckerhaltiger Urine durch Bleiessig eine Ausfällung von Zucker

1) Zeitschr. f. anal. Chemie 1873. 296. 
nicht zu befürchten ist. Dennoch ziehe ich dieser Methode die Anwendung von Bleizackersolution vor, da ich selbst bei Zusatz gleicher Vol. einer solchen zu allen oben aufgeführten Harnen weder bei sofortiger Beobachtung, noch auch nach 24 Stunden, eine stärkere Abnahme der Rotation erkennen konnte, als der eingetretenen Vergrösserung des Flüssigkeitsquantums genau entsprach.

Ich bemerke noch, dass ich mich der Ausfällung der Urochloralsäure durch Bleiessig häufig mit Erfolg bedient habe, um dic Harne eines Individuums auf Zuckergehalt zu untersuchen, welches früher Urine mit $4 \%$ Zucker ausgeschieden hatte, aber nach einer längere Zeit eingehaltenen, entsprechenden, Diät solche von sich gab, die nicht oder schwach nach rechts oder links drehten. Der Patient nahm jeden Abend $3 \mathrm{~g}$. Chloralhydrat ein.

Wegen der dargelegten Mängel der Ausfällung der Urochloralsäure aus Harnen durch Bleiessig wäre es sehr erwünscht, wenn dieser Methode eine andere bessere Art der Bestimmung von Zucker neben Urochloralsäure substituirt werden könnte; wozu allerdings vorläufig wenig Aussicht vorhanden zu sein scheint. Die Fehling'sche Titrirmethode wird diesem Zwecke in vielen Fällen entsprechen, da, wie ich später darthun werde, die reducirende Wirkung der Urochloralsänre eine weit schwächere ist als die der Glucose und dieselbe daher bei hinreichend hohen Zuckergehalten von Harnen durch geeignete Dilution aufgehoben werden kann, ohne dass die Bestimmung der Glucose dadurch an Genauigkeit einbüsst. Bei den schwach zuckerhaltigen Urinen von Diabetikern, die sich in der Besserung befinden, wird die Fehling'sche Methode nicht zu dem bezeichneten Ziele führen, da solche Exorete, abgesehen davon, dass dieselben sich an und für sich nicht selten zur Titration wenig eignen, bei einem etwaigen Gehalte an Urochloralsäure häufig nicht bis zur Unschädlichmachung der letzteren werden verdünnt werden können, ohne dass dadurch das Résultat der Zuckerbestimmung beeinflusst würde.

Beeinflussung der Prüfung von Harnen a uf Zucker nach den gebräuchlichsten einfachen Methoden durch einen Gehalt an Urochloralsäure.

Die Veranlassung zu den angestellten Untersuchungen lag in der Mittheilung von Musculus und v. Mering, dass die Uro- 
chloralsäure Kupferoxyd, Wismuthoxyd und Indigo reducire und sich beim Erhitzen mit Kalilauge unter Verbreitung von Caramelgeruch bräune.

Da nach Seegen die Trommer's che Probe zur Erkennung des Zuckers in Harnen die geeignetste ist, so habe ich eine grosse Zahl von Chloralharnen hinsichtlich ihres Verhaltens bei jener Probe untersucht. Ich fand, dass die Mehrzahl derselben kräftige Reductionen bewirkte mit nachfolgender Ausfällung von Kupferoxydulhydrat, weit seltener von Oxydul; die Stärke der Reactionen stand meistens im Verhältnisse zu den Rotationen der Excrete. In der Regel erfolgte die Hauptreaction erst nach Eintritt des Siedens oder bei nachherigem Stehen der gekochten Flüssigkeiten.

Auch die durch Thierkohle entfärbten Harne und hänfig das erste Waschwasser der Kohle, manchmal sogar noch das zweite, bewirkten mehr oder weniger intensive Reductionen mit Ausscheidung von Kupferoxydulhydrat resp. Oxydul. Dies schien leicht erklärlich zu sein, da nach früher mitgetheilten Versuchen Chloralharnen bei der Filtration durch Knochenkohle nicht selten Urochloralsäure entzogen wird. Der in die Filtrate übergegangene Rest der Säure, sowie die der Kohle anhaftende Quantität derselben war hinreichend, um die beschriebenen Reactionen hervorzubringen. Ich bemerke, dass die Personen, deren Harne ich untersuchte, ohne Chloralgenuss solche ausgeschieden hatten, die nur in normaler Weise reducirten, sowie dass bei Unterbrechung der Einnahme nach einiger Zeit die Urine der Individuen nur mehr die normale Reductionsfähigkeit zeigten. Da manche Chloralharne von schwacher Rotation bedeutend stärker reducirten als andere stärker rotirende, so konnte aus der alleinigen Untersuchung von Chloralharnen kein scharfes Urtheil über die Beeinflussung der Zuckerreactionen von Seiten der Urochloralsäure gewonnen werden. Jenes auffallende Verhalten einiger Chloralharne konnte darin begründet sein, dass die untersuchten Chloralharne sämmtlich von Geistes - und körperlich Kranken herstammten, deren Harne nach Abeles ${ }^{1}$ nicht selten geringe Quantitäten Zucker enthalten sollen. Natürlich kann dieser Umstand nicht die in der Regel stärkere reducirende Wirkung der Harne nach als vor Chloralgenuss

1) Wicner medic. Wochensehr. 1874. 
erklären. Das erwähnte stärkere Reductionsvermögen mancher Chloralharne schwacher Rotation gegenüber weit stärker drehenden mag mitunter seinen Grund darin finden, dass die Quantitäten Chloral, welche unverändert in den Harn übertreten, vielleicht in verschiedenen Fällen erheblich untereinander varïren (?).

Eine 0,2 procentige Solution des Hypnoticums rief bei der Fehling'schen Probe schon kurz vor, eine 0,1 procentige erst bei Beginn des Kochens eine gelbe Fällung hervor, während eine 0,05 procentige Lösung auch bei wiederholtem Aufkochen und nachfolgendem längerem Stehen keine Farbenänderung der alkalischen Kupfersulfatsolution mehr zu Wege brachte. $O b$ ein Gehalt von Harnen an Chloral wirklich im Stande sein kann, einen Gehalt an Zucker vorzutäuschen, kann vorläufig nicht entschieden werden, da nichts Näheres über die Mengen des Hypnoticums bekannt ist, welches durch die Nieren unverändert ausgeschieden wird, wenn es anch nach den bisher darüber angestellten Untersuchungen wahrscheinlich ist, dass dieselben nur sehr geringe sein werden.

Um mir eine Ansicht darüber zu bilden, in wie weit die Gehalte von Chloralharnen an Urochloralsäure das Resultat der Zuckerprüfung beeinflussen können, habe ich vergleichende Versuche über das Verhalten der beiden Körper bei den oben genannten Zuckerproben angestellt. Ich Jasse dieselben auf zwei Tabellen folgen und zwar zunächst die Versuche mit Urochloralsäure. Zu jeder der Proben dienten 1,5 C.C. der Solutionen; die einzelnen Versuche wurden genau nach Neubauer's Vorschrift executirt.

Ein Reductionsvermögen der Urochloralsäure für basisches Wismuthnitrat habe ich bei einer Lösung von urochloralsaurem Kalium mit der Rotation -2 nicht erkennen können, auch nicht bei lange Zeit fortgesetztem Kochen. $O b$ dieses negative Resultat in der zu geringen Concentration der Lösung oder darin seinen Grund hatte, dass mir ein reineres Präparat zu Gebote stand als den H. H. Musculus und v. Mering lasse ich vorläufig unentschieden, jedenfalls aber zeigen meine Versuche, dass bei der Untersuchung von Harnen, die nach Einnahme von $2-3$ g. Chloralhydrat gelassen werden, die Vortäuschung eines Zuckergehaltes durch jene Säure nicht zu befürchten ist, wenn man sich der Böttger'schen Probe bedient. Sollen auch kleine Mengen Zucker in Harne berücksichtigt werden, so kommt die Wismuthprobe wegen ihrer geringen 
Verhalten wässeriger Lösungen von urochloralsanrem Kalium bei den üblichen einfachen Zuckerproben:

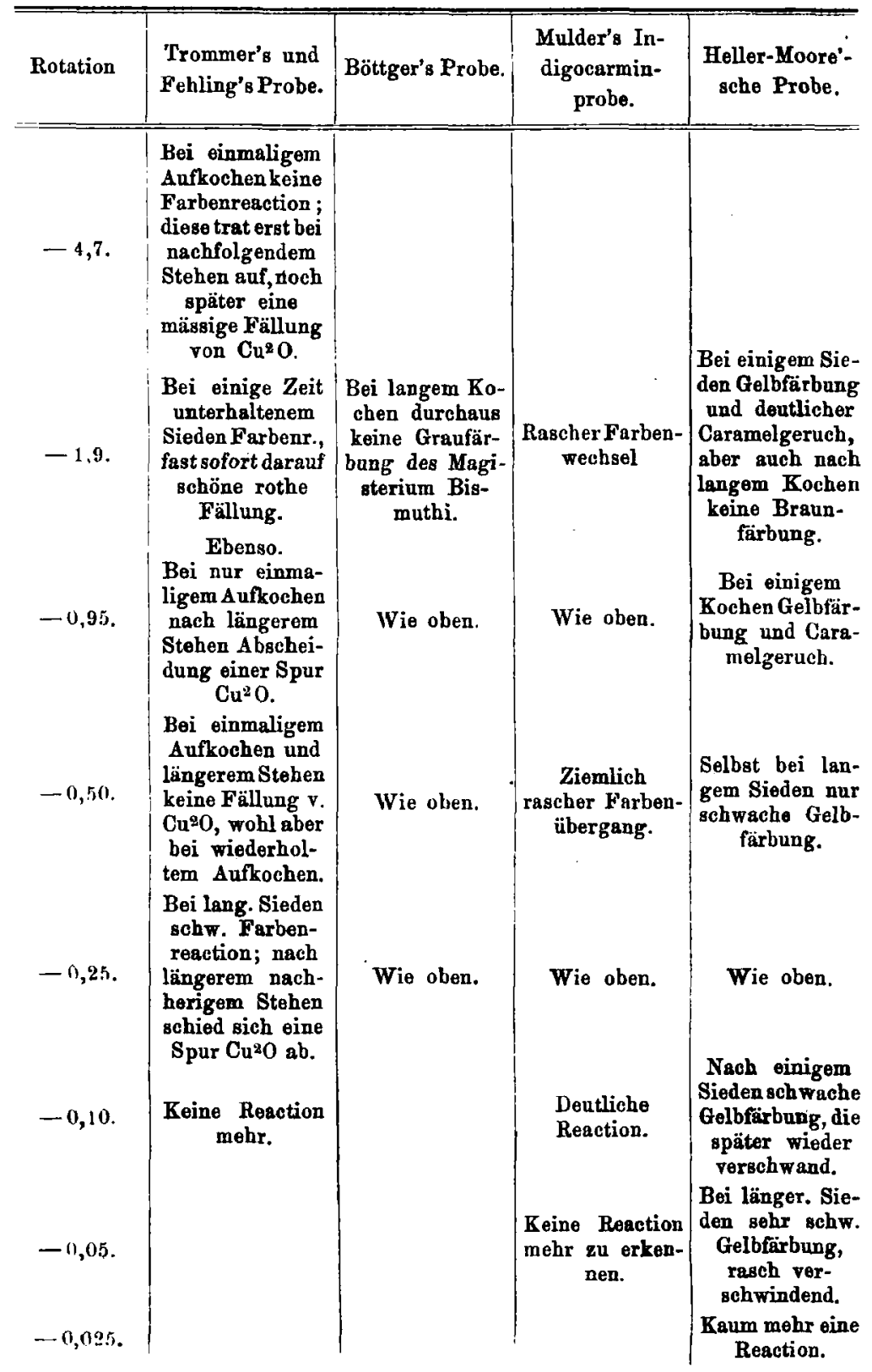


A. Bornträger, Ueber die Entslehung der Urochloralsäure ete.

Verhalten von Glucosesolutionen beiden gewöhnlichen einfachen Zuckerproben:

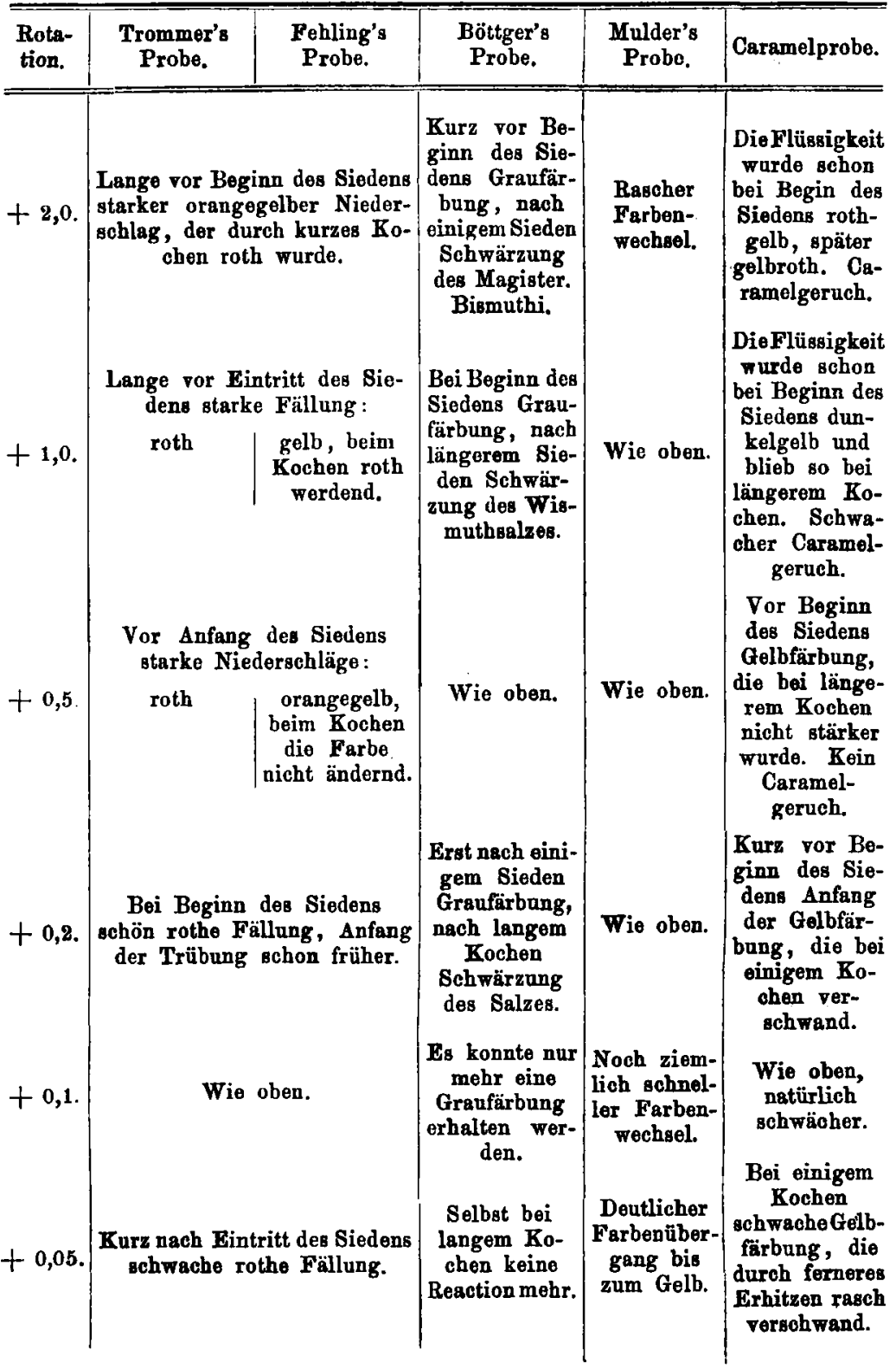




\begin{tabular}{|c|c|c|c|c|c|}
\hline $\begin{array}{l}\text { Rota- } \\
\text { tion. }\end{array}$ & $\begin{array}{c}\text { Trommer's } \\
\text { Probe. }\end{array}$ & $\begin{array}{c}\text { Fehling's } \\
\text { Probe. }\end{array}$ & $\begin{array}{c}\text { Böttger's } \\
\text { Probe. }\end{array}$ & $\begin{array}{c}\text { Mulder's } \\
\text { Probe. }\end{array}$ & Caramelprobe. \\
\hline$+0,02$ & $\begin{array}{r}\text { Einige Zeit na } \\
\text { Siedens minin } \\
\text { schlag v }\end{array}$ & $\begin{array}{l}\text { Eintritt des } \\
\text { aler Nieder- } \\
\text { n } \mathrm{Cu}^{2} \mathrm{O} \text {. }\end{array}$ & & $\begin{array}{l}\text { Langsame } \\
\text { Farben- } \\
\text { wandlung } \\
\text { big zum } \\
\text { Gelb. }\end{array}$ & $\begin{array}{l}\text { Bei Siedhitze } \\
\text { ganz schwache } \\
\text { Gelbfärbung, } \\
\text { die bei kurzem } \\
\text { Stehen ver- } \\
\text { schwand. }\end{array}$ \\
\hline$+0,005$ & $\begin{array}{c}\text { Schwer zu } \\
\text { entscheiden } \\
\text { wegen des un- } \\
\text { gelösten Ku- } \\
\text { pferoxyds, } \\
\text { welches die } \\
\text { Reaction ver- } \\
\text { deckte. }\end{array}$ & $\begin{array}{c}\text { Kurz nach } \\
\text { Eintritt des } \\
\text { Kochens } \\
\text { Farbenr., so- } \\
\text { dann gelbe } \\
\text { Trübung, nach } \\
\text { längerem Ste- } \\
\text { hen geringes } \\
\text { Sediment von } \\
\text { Cu² O }^{2}\end{array}$ & & & \\
\hline$+0,001$. & Ebenso. & $\begin{array}{l}\text { Nach langem } \\
\text { Kochen schw. } \\
\text { Farbenreac- } \\
\text { tion, bei län- } \\
\text { gerem Stehen } \\
\text { minimale Trü- } \\
\text { bung obne } \\
\text { Sedimentiren. }\end{array}$ & & & \\
\hline $\begin{array}{c}+0,0005 \\
\text { und } \\
0,0001 .\end{array}$ & Ebenso. & $\begin{array}{c}\text { Bei langem } \\
\text { Kochen nur } \\
\text { Farben- } \\
\text { reaction. }\end{array}$ & & & \\
\hline
\end{tabular}

Empfindlichkeit bekanntlich nicht in Betracht, dagegen kann dieselbe bei mässigen Zuckergehalten von Harnen, bei deren Prüfung allerdings auch die Trommer'sche Probe kaum falsch zu deutende Reactionen ergiebt, zur Bestätigung des Resultates der letzteren Probe dienen.

Was das Verhalten der Glucose und der Urochloralsäure gegen Kupferoxyd anbelangt, so wirkt erstere schneller und noch bei weit stärkerer Verdünnung ihrer Lösungen reducirend. Wälrend nämlich 0,1 procentige Glucoselösungen schon zu Beginn des Siedens eine charakteristische rothe Fällung und etwas früher eine Trübung ergaben, bewirkte eine Lösung von urochloralsaurem Kalium mit der Drehung - 4,7 erst bei einige Zeit fortgesetztem Sieden oder bei längerem Stehen des nur einmal aufgekochten Gemisches eine Farbenveränderung der Fehling'schen Probeflüssig- 
keit. Es kann somit eine Verwechselung selbst grosser Mengen Urochloralsäure mit nur mässigen Mengen Glucose nicht wohl stattfinden. Bei Chloralharnen, die grosse Quantitäten solcher Stoffe enthalten, die Kupferoxydul oder dessen Hydrat in Lösung halten, kann selbstredend nur aus dem raschen Verlaufe der Farbenveränderung bei der Kupferoxydprobe auf einen Gehalt an Zucker, der $0,2 \%$ übersteigt, geschlossen werden.

Selbstredend können kleine Quantitäten Zucker und Spuren desselben, deren continuirliches Vorkommen im Harne nach Seegen ${ }^{1}$ ebenfalls der Ausdruck eines pathologischen Vorganges im Organismus ist, neben grösseren Mengen Urochloralsäure in der angegegebenen Weise nicht nachgewiesen werden, da jene die Reduction des Kupferoxyds ebenfalls nar langsam bewirken.

Von der Anstellung der Kupferoxydprobe in der Kälte ist bei der Untersuchung von Chloralharnen auf geringe Quantitäten und Spuren von Zucker ebenfalls kein günstiger Erfolg zu hoffen, da 0,05 procentige Glucosesolutionen nach Seegen's Angaben, die ich als richtig erkannt habe, keine Reaction auf Kupferoxyd in der Kälte mehr äussern und Chloralhydrat sich in Beziehung auf diese Reaction dem Zucker ganz gleich verhält, indem eine 0,1 procentige Lösung jener Substanz noch Kupferoxyd in der Kälte reducirt, nicht aber mehr eine 0,05\% enthaltende.

Bei der Untersuchung von Chloralharnen ist, wie in den meisten anderen Fällen, die von Seegen modificirte Trommer'sche Probe der einfachen entschieden vorzuziehen, denn einerseits gestattet dieselbe noch den Nachweis sehr kleiner Quantitäten Zncker im Harne, andererseits wird, da nach den von mir mitgetheilten Versuchen Thierkohle aus Urinen häufig kein, manchmal etwas urochloralsaures Kalium aufnimmt, das farblose Filtrat zwar eine ebenso oder fast so starke Reduction bewirken als der unveränderte Harn, das erste Waschwasser wird wegen der geringen reducirenden Kraft der Urochloralsäure keine oder doch nur eine unbedeutende Fällung von Kupferoxydul veranlassen, während den späteren Waschflüssigkeiten diese Fähigkeit mit ziemlicher Gewissheit abzusprechen sein wird. Diese Vermuthung wurde durch die Resultate der Untersuchungen einer Anzahl von Chloralharnen bestätigt, von welchen ich nur die folgenden anführen will.

1) Diabetes mollitus 1875.194. 
Erste Versuchsreihe:

Hellgelber Morgenurin, Rotation - 0,8, Pat. hatte am vorhergegangenen Abend $3 \mathrm{~g}$. Chloralhydrat erhalten.

\section{Trommer's Probe:}

Unveränderter Harn: Nach einmaligem Aufkochen beim Stehen Fällung von Kupferoxydulhydrat.

Nach Verdünnung mit der dreifachen Wassermenge: Bei wiederholtem Aufkochen keine Fällung von Oxydul, sondern nur eine Farbenreaction.

Entfärbter Harn: Beim Stehen nach einmaligem Aufkochen starker gelber Niederschlag.

Erstes Waschwasser: Ebenfalls bei längerem Stehen der cinmal aufgekochten Mischung Abscheidung von Oxydulhydrat.

Zweites Waschwasser: Bei wiederholtem Aufkochen und nachfolgendem längerem Stehen keine Abscheidung mehr, sondern nur eine Farbenreaction.

\section{Zweite Versuchsreihe.}

Normal gefärbter Frühharn, Drehung - 0,75, Pat. hatte $2 \mathrm{~g}$. Chloralhydrat am vorhergehenden Abende eingenommen.

\section{Trommer's Probe:}

Unveränderter Harn: Bei einmaligem Aufkochen Farbenreaction, beim Stehen nach ca. 5 Minuten missfarbige Fällung.

Entfärbter Urin (Rotation - 0,70): Bei einmaligem Aufkochen Farbenreaction, bei nachherigem Stehen in wenigen Minuten Trübung, sodann gelbe Fällung, die bei nochmaligem Sieden zunahm und orangefarbig wurde.

Erstes Waschwasser: Einige Zeit nach einmaligem Aufkochen orangegelbe Fällung, von oben nach unten auftretend.

Zweites Waschwasser: Selbst bei mehrmaligem Kochen und nachfolgendem längerem Stehen keine Abscheidung mehr.

\section{Dritte Versuchsreihe.}

Normal aussehender Morgenurin, spec. Gew. 1,024, Rotation $-0,75$, nach Einnahme von $4 \mathrm{~g}$. Chloralhydrat gelassen.

\section{Trommer's Probe:}

Unveränderter und entfärbter Harn: Einige Zeit nach dem Aufkochen gelbe Fällung. 
Erstes Waschwasser: Erst nach wiederholtem Kochen schöner orangefarbiger Niederschlag.

Zweites Waschwasser: Bei gleicher Behandlung nur eine Farbenreaction.

Den aufgeführten Harnen verhielten sich eine Anzahl gleich, stärker und schwächer rotirender ähnlich. Ich bemerke noch, dass bei schwach rotirenden Chloralharnen häufig schon das erste Waschwasser keine Abscheidung von Kupferoxydul oder dessen Hydrat mehr zu Wege brachte. Das Reductionsvermögen stark urochloralsäurehaltiger Urine konnte durch starke Verdünnung mit Wasser aufgehoben werden, z. B. durch 10 fache, nicht durch 5 fache, Dilution bei einem Harne der Rotation - 1,05. Diese Thatsache spricht zu Gunsten der S. 449 ausgesprochenen Vermuthung, dass in ziemlich stark zuckerhaltigen Harnen diese Substanz sich durch die Fehling'sche Titrirmethode werde bestimmen lassen, auch wenn die Excrete urochloralsäurehaltig sein sollten.

Hinsichtlich des schon erwähnten abweichenden Verhaltens einer grossen Anzahl Chloralharne, unter welchen sich auch viele schwach rotirende befanden, von dem oben skizzirten Verhalten, indem auch das zweite, mitunter sogar die folgenden Waschwässer noch eine Abscheidung von Kupferoxydul oder dessen Hydrat bewirkten, verweise ich auf das S. 450 und 451 Gesagte. Für mich liegt in den dort dargelegten Verhältnissen eine Warnung davor, aus dem scheinbar positiven Resultate der Seegen'sohen Probe in Chloralharnen direct den Schluss auf einen Zuckergehalt derselben zu ziehen, wenn ich auch wegen der bedeutenden Schwierigkeiten der Ausführung nicht streng den Nachweis geliefert habe, dass jene Chloralharne, welche stärker reducirten, als ihren Urochloralsäuregehalten entsprechen konnte, frei von kleinen Zuckermengen waren. In den Fällen, in welchen das zweite Waschwasser der zur Entfärbung von Chloralharnen benutzten Thierkohle keine Reduction mehr bewirkt, kann das Fehlen von Glucose in Quantitäten von $0,05 \mathrm{~g}$. an aufwärts gefolgert werden, indem nach Seegen noch das vierte Waschwasser von Thierkohle, die zur Entfärbung normaler Harne benutzt wurde, denen jene Zuckermenge zugesetzt worden war, geringe Mengen Kupferoxydul zư bilden vermoohte. Diese grosse Empfindlichkeit der Seegen'schen Prïfungsmethode ist unschwer $\mathrm{zu}$ verstehen, da Trommer ${ }^{1}$ durch

1) Annal. d. Chem. u. Pharm. Bd. 39, 360. 
eine Lösung mit 0,00001 Theilen Traubenzucker noch einen deutlichen rothen oder gelben Niedersohlag eintreten seh; ich erhielt den auf S. 454 mitgetheilten Versuchen zu Folge noch einen minimalen Niederschlag mit $0,000015 \mathrm{~g}$. Glucose, gelöst in 1,5 C.C. Wasser.

Ich will mich nunmehr der Besprechung des Verlaufes der Caramelprobe in urochloralsäure- und glucosehaltigen Harnen zuwenden. Eine 0,5 procentige Traubenzuckersolution ergab beim Erwärmen mit Natronlauge zwar schon vor Eintritt des Siedens eine Gelbfärbung, da diese aber bei längerem Kochen nicht in Rothgelb oder Rothbraun überging und nicht von Caramelgeruch begleitet wurde, so war dieselbe nioht mehr als eine charakteristische Reaction anzusehen. Ueberdies kann ein Gehalt von Urinen an Urochloralsäure zu Täuschnngen bei dieser Probe Veranlassung geben, da nach den mitgetheilten Versuchen eine 0,5procentige Zuckersolution eine nicht stärkere, wenn auch früher erfolgende, Farbenveränderung ergab als eine Lösung von urochloralsaurem Kalium mit der Rotation - 1,0, welch' letztere im Unterschiede von jener sogar Caramelgeruch entwickelte. Ich darf nicht unerwähnt lassen, dass Fürbringer noch mit einer 0,2\% Glucose enthaltenden Flïssigkeit Caramelgeruch erhalten zu haben angiebt.

Was schliesslich die Indigo- oder Mulder'sche Probe anbetrifft, welche ebenso wie die Caramelprobe beim Aufsuchen kleiner Zuckermengen in Harnen nicht in Betracht kommt, so würde diese Bestrebung weiter auch noch durch einen gleichzeitigen Gehalt der betreffenden Urine an Urochloralsäure vereitelt werden, da diese noch in Lösungen der Rotation $-0,25$ eine ziemlich rasch verlaufende, in solchen mit der Drehung - 0,10 noch eine deutliche, Farbenumwandlung bewirkt. Eine 0,02 procentige Glucosesolution ergiebt bei der Mulder'schen Probe nur noch eine schwache Reaction.

Ich fasse die Resultate der in dem letzten Capitel angestellten Betrachtungen dahin zusammen, dass die Trommer'sche (resp. Fehling'sche) und die Böttger'sche Probe den Nachweis mässiger Mengen Glucose (ron $0,1-0,2 \%$ aufsteigend) neben Eiemlich viel Urochloralsäure (entsprechend der Rotation -2,0) ermöglichen, dass aber die Auffindung von kleinen Quantitäten und Spuren des Kohlehydrates neben viel Urochloralsëure mit Hülfe jener Proben nicht zu erreichen ist. Die Seegen'sche 
Probe gestattet noch den Nachweis von $0,05 \%$ Glucose neben viel Urochloralsäure (der Rotation - 1,0 entsprechend, wie nachgewiesen wurde, ob auch stärkeren, wurde nicht untersucht, ist aber anzunehmen).

Die Caramel- und die Indigoprobe sind zum Nachweise kleiner Mengen Glucose neben Urochloraleäure weit weniger zu empfehlen als die vorher erwähnten Methoden.

In wie weit diese Verhältnisse durch den Gehalt von Chloralharnen an unverändertem Chloral eine Umgestaltung erfahren, ist nicht mit Sicherheit festgestellt. Hinsichtlich der Beurtheilung und Verwerthung der bei der Seegen'schen Prüfungsmethode mit jenen Excreten erhaltenen Reactionen verweise ich auf die S. $455 \mathrm{ff}$. gemachten Ausführungen.

Aus Vorstehendem ergiebt sich die Schwierigkeit, die Frage, ob die Harne eines Patienten, der Chloral eingenommen hat, zuckerhaltig sind oder nicht, in allen Fällen streng im Sinne Seegen's zu beantworten.

Vorstehende Arbeit habe ich während meiner Anstellung als Chemiker der Maison de Santé in Schöneberg ausgeführt und danke ich deren Director Herrn Geh. Sanitätsrath Dr. Ed. Levinstein an dieser Stelle bestens für die gütige Ueberlassung der erforderlichen Materialien.

\section{Jodoform aus Mothylalkobol.}

Von E. Biltz in Erfurt.

In dem kürzlich ausgegebenen Märzhefte des Archivs findet sich Seite 232 eine aus dem Bulletin de la société chimique ${ }^{1}$ in amerikanische Blätter übergegangene Angabe von Guyard, nach welcher eine Auflösung von Jod in Jodkalium ein Reagens auf Methylalkohol sein soll (durch Jodoformbildung), während Aethylalkohol damit keine Fällung bewirke.

1) A. Guyard, Bulletin de la société chimique de Paris 1879. T. 31. p. 297 u. f. Arch. d. Pharm. 1879. Bd. 215. S. 176, u. 1880. Bd. 216. 8. 232. 